\title{
Predictive biomarkers for cancer immunotherapy with immune checkpoint inhibitors
}

\author{
Rilan Bai, Zheng LV, Dongsheng Xu and Jiuwei Cui
}

\begin{abstract}
Although the clinical development of immune checkpoint inhibitors (ICIs) therapy has ushered in a new era of antitumor therapy, with sustained responses and significant survival advantages observed in multiple tumors, most patients do not benefit. Therefore, more and more attention has been paid to the identification and development of predictive biomarkers for the response of $\mathrm{ICls}$, and more in-depth and comprehensive understanding has been continuously explored in recent years. Predictive markers of ICls efficacy have been gradually explored from the expression of intermolecular interactions within tumor cells to the expression of various molecules and cells in tumor microenvironment, and been extended to the exploration of circulating and host systemic markers. With the development of high-throughput sequencing and microarray technology, a variety of biomarker strategies have been deeply explored and gradually achieved the process from the identification of single marker to the development of multifactorial synergistic predictive markers. Comprehensive predictive-models developed by integrating different types of data based on different components of tumor-host interactions is the direction of future research and will have a profound impact in the field of precision immuno-oncology. In this review, we deeply analyze the exploration course and research progress of predictive biomarkers as an adjunctive tool to tumor immunotherapy in effectively identifying the efficacy of $\mathrm{ICls}$, and discuss their future directions in achieving precision immuno-oncology.
\end{abstract}

Keywords: Neoplasm, Immune checkpoint inhibitor, Predictive biomarker, Tumor mutation burden, Programmed death ligand-1

\section{Background}

Immune checkpoint inhibitors (ICIs) therapy has ushered in a new era of anti-tumor therapy, with sustained responses and significant survival advantages observed in multiple tumors. Anti-programmed cell death-1/programmed cell death-ligand 1 (PD-1/PD-L1) antibody has been approved for second-line or first-line treatment in a variety of malignant neoplasms, including melanoma, lung cancer, renal cell carcinoma (RCC), head and neck squamous cell carcinoma (HNSCC) and gastroesophageal

\footnotetext{
* Correspondence: cuijw@jlu.edu.cn

Cancer Center, the First Hospital of Jilin University, 71 Xinmin Street, Changchun, Jilin 130021, China
}

cancer [1, 2]. However, despite the breakthrough in clinical treatment with ICIs, most patients do not benefit. Pembrolizumab or nivolumab has an objective response rate (ORR) of $40-45 \%$ in first-line melanoma and $20 \%$ in second-line non-small cell lung cancer (NSCLC) [3-5]. Therefore, in recent years, more and more attentions have been paid to the identification and development of predictive biomarkers for the efficacy of ICIs, and more indepth and comprehensive understanding has also been obtained in recent years, including new data on biomarkers of tumor genome and neoantigen, tumor immune microenvironment phenotype, liquid biopsy biomarkers, host-related factors and all of which have 
made many new advances in the corresponding fields. With the development and continuous improvement of multiplex immunohistochemical technology, highthroughput sequencing and microarray technology, a variety of biomarker strategies have emerged and gradually realized the process from the identification of single marker to the development of multifactorial synergistic predictive markers. The development of predictive biomarkers contributes to revealing the therapeutic mechanisms of ICIs and the interaction mechanisms between tumor and host immunity, achieving decision-making of individualized anti-tumor immunotherapy, monitoring efficacy and disease development, guiding clinical trial design, as well as for further understanding of drug resistance mechanisms and tumor prognosis. In this review, we deeply analyze the exploration course and research progress of predictive biomarkers as an adjunctive tool to tumor immunotherapy in effectively identifying the efficacy of ICIs. It should be pointed out here that when reading and collating, we try to read and include all the relevant articles. In the process of selecting articles, we include the authoritative articles published in high-level journals or the latest research results, and objectively describe and analyze their roles in this field, as well as discuss the reasons that different research results may be involved.

\section{Advances of multiple predictive biomarkers to ICls efficacy}

\section{(i). Tumor genome and neoantigen biomarkers}

\section{Tumor mutation burden}

Significant correlations between high tumor mutation burden (TMB) and response to ICIs have been reported in several cancer types [6], including urothelial carcinoma [7], small cell lung cancer (SCLC) [8], NSCLC [9-11], melanoma [12], and human papilloma virus (HPV)-negative HNSCC [13]. A meta-analysis of 27 cancer types showed that the mean response rate was positively correlated with $\log (\mathrm{TMB})$ [14]. The National Comprehensive Cancer Network (NCCN) guidelines have adopted TMB as the recommended test for patients with NSCLC receiving immunotherapy. Although the results in some clinical studies of RCC [15], HPV-positive HNSCC [13], and melanoma receiving anti-PD-1 after recurrence [16] showed that $\mathrm{TMB}$ alone also did not clearly distinguish responders and predict OS, it is still exciting that multiple studies in the 2020 American Society of Clinical Oncology (ASCO) meeting have confirmed the predictive value of TMB in immunization or combination therapy (KEYNOTE-061 study [17, 18], CONDOR study [19], EAGLE study [20], EPOC1704 study [21], etc.), consolidating its status of TMB as an independent predictor. And in April 2020, the
U.S. Food and Drug Administration (FDA) prioritized the approval of TMB as a companion diagnostic biomarker for pembrolizumab.

Nonetheless, the cut-off values of TMB were defined differently across studies and assay platforms, such as atezolizumab $>16 \mathrm{mt} / \mathrm{Mb}$ in urothelial cancer, pembrolizumab > $23.1 \mathrm{mt} / \mathrm{Mb}$ in NSCLC, and atezolizumab $\geq 13.5, \geq 15.8$, or $\geq 17.1 \mathrm{mt} / \mathrm{Mb}$ in NSCLC [22-25], and nivolumab plus ipilimumab $\geq 10 \mathrm{mt} / \mathrm{Mb}$ in NSCLC [10, 26], which needs further study to confirm the optimal cut-off value in different tumors. Moreover, the NGS panels have approved by the FDA that can be used to estimate TMB include the MSK-IMPACT and FoundationOne CDx panel, the detection results of which are highly consistent with whole exome sequencing (WES) $[11,27]$, and other solutions are under development. A study detecting TMB (cut-off value at $20 \mathrm{mt} / \mathrm{Mb}$ ) in 4064 NSCLC patients with the FoundationOne platform containing a 395 gene panel found that compared with TMB-L patients, overall survival (OS) and DCR was significantly improved in TMB-H patients treated with anti-PD-1/L1 drug [11]. Both WES and targeted NGS (a 422-cancer-gene panel) performed in 78 patients with NSCLC treated with anti-PD-1/L1 demonstrated that TMB-H population has a significantly better durable clinical benefit (DCB) and progression-free survival (PFS) [27]. These findings demonstrate the feasibility of comprehensive genomic profiling (CGP), but the design of optimal next generation sequencing (NGS) panel that is more accurate, comprehensive and cost-effective is still not clear. In addition, given that bTMB was identified as a predictor of PFS but failed to differentiate patients with OS benefits, researchers consider the need to explore other more precise factors, e.g. allele frequency (AF). A study that developed a new bTMB algorithm in two independent cohorts (POPLAR and OAK) showed that modified bTMB, low AF bTMB (LAF-bTMB, mutation counts with an $\mathrm{AF}<5 \%$ ), was significantly associated with favorable $(\mathrm{HR}=0.70,95 \% \mathrm{CI} 0.52-0.95, p=$ 0.02 ), PFS (HR $=0.62,95 \% \mathrm{CI} 0.47-0.80, p<0.001$ ), and ORR $(\mathrm{p}<0.001)$ after immunotherapy, but required to be prospectively validated [28]. Finally, static biomarkers are insufficient to accurately predict response due to the complexity of tumor-immune interactions. A recent analysis of tumor genome-wide dynamic detection in pretreatment and on-treatment melanomas found that pretreatment TMB was only associated with OS in untreated patients, while early (4-week) on-treatment change in TMB $(\triangle \mathrm{TMB})$ was strongly associated with anti-PD-1 response and OS in the entire cohort [16]. The detection of $\triangle \mathrm{TMB}$ is helpful for early evaluating the response to therapy of patient, but its clinical usability limited by the difficulty in obtaining tissue samples and high price, while liquid biopsy discussed below might better. 
In addition, epigenetic changes are associated with TMB. The latest study investigated the association between TMB and DNA methylation (DNAm) to explore potential complimentary biomarkers for NSCLC immunotherapies. The results showed that high TMB NSCLCs had more DNAm aberrance and copy number variations (CNVs), showing certain value in predicting efficacy, such as HOX gene methylation status and TMB [29] Thus, the correlated exploration of epigenetics has attracted more attention in recent years, and liquid biopsy-based epigenetic studies may become a future research direction. Exploration in Chinese NSCLC patients showed that NSCLCs with high TMB had DNAm aberrance and CNVs. Some insertion and deletion (indel) mutations can lead to frameshifts and more immunogenic neoantigens [30]. In the pan-cancer analysis of 19 cancer types evaluated in The Cancer Genome Atlas (TCGA), RCC had the highest indel mutation load, and frameshift indel mutations were found to produce three times more candidate neoantigens per mutation than nonsynonymous single nucleotide variants (nsSNVs) [30]. Somatic copy number alterations (SCNAs) are another feature of the genomic landscape of tumors, and pan-cancer TCGA analysis revealed an inverse correlation between SCNAs at the single-arm or whole chromosome-level and immune infiltration in 10 tumor types tested [31], and this result was subsequently replicated in a larger study of TCGA [32].

\section{DNA damage response pathways}

Genetic variation involved in DNA mismatch repair (MMR) pathway can lead to microsatellite instability (MSI), a specific type of high TMB tumors, and increased numbers of $\mathrm{CD}^{+}$tumor infiltrating lymphocytes (TILs), PD-1 $1^{+}$TILs, and indoleamine 2,3-dioxygenase $(\mathrm{IDO})^{+}$tumor cells have been shown in MMR deficiency (dMMR) colorectal cancer [33]. Recently, five clinical trials (Keynote-016, 164, 012, 028, 158) including multiple tumor types have shown that patients with dMMR/MSI$\mathrm{H}$ can achieve durable responses to pembrolizmab. Based on this, pembrolizumab is approved by the U.S. FDA for the treatment of any advanced solid tumor with $\mathrm{dMMR} / \mathrm{MSI}-\mathrm{H}$, and nivolumab in combination with ipilimumab has also shown promising response in dMMR/ MSI-H colorectal cancer [34]. In addition, dMMR can also cause mutations in the DNA polymerase gene epsilon/delta 1 (POLE/POLD1), increasing the mutation load and neoantigen load. Analysis of POLE/POLD1 mutations in 47,721 patients with different cancer types showed that patients with these mutations had significantly higher TMB and OS. Therefore, it may be an independent risk factor and prognostic marker for identifying patients who benefit from ICIs [35]. In addition, pathways of base excision repair (BER), homologous recombination repair (HRR), MMR in the DNA damage response (DDR) signaling network contribute more significantly to TMB or neoantigens, which have the highest levels when co-mutated [36]. It had been identified that co-mutations in the DDR pathways of HRR and MMR or HRR and BER, defined as co-mut ${ }^{+}$, are associated with increased levels of TMB, neoantigen load and immune gene expression signatures. Co-mut ${ }^{+}$ patients showed a higher ORR and longer PFS or OS, indicating that co-mut can be used as predictors of response to ICIs and provide a potentially convenient method for future clinical practice [36].

\section{Specific mutated gene pathways in tumor cells}

It is worth noting that alterations of signaling pathways in tumor cells affect the responsiveness to immunotherapy. Patients with mutations in the interferon (IFN)- $\gamma$ pathway genes, IFNGR1/2, JAK1/2, and IRF1, are poorly responsive to ICIs treatment and confer resistance [37]. A study found that in patients receiving immunotherapy, tumor cells can downregulate or alter IFN- $\gamma$ signaling pathways such as loss-of-function alleles of genes encoding for JAK1/2, and changes in STAT1 to escape the influence of IFN- $\gamma$ [38], resulting in poor efficacy and resistance. Recent studies suggest that inactivating mutations in a mammalian analog of the chromatin remodeling SWI/SNF complex and unique genes of the PBAF complex (Pbrm1, Arid2, and Brd7) lead to sensitivities to ICIs $[39,40]$. Loss of function of the PBAF complex increased chromatin accessibility to transcription regulator elements of IFN- $\gamma$-inducible genes within tumor cells, and subsequently increased production of CXCL9/ CXCL10 chemokines, leading to more efficient recruitment of effector $\mathrm{T}$ cells into tumors [41]. In human cancers, expression of Arid2 and Pbrm1 are related to expression of $\mathrm{T}$ cell cytotoxicity genes, which confirmed in Pbrm1-deficient murine melanomas with strongly infiltrated by cytotoxic $\mathrm{T}$ cells and responsive to immunotherapy [15, 41]. In addition, double-stranded RNA (dsRNA) editing enzyme adenosine deaminase acting on RNA (ADAR1) protein can block the IFN- $\gamma$ signaling pathway and lead to poor ICIs efficacy and resistance. Loss of function of ADAR1 in tumor cells can reduce Ato-I editing of interferon-inducible RNA species and lead to dsRNA ligand sensing by PKR and melanoma differentiation-associated protein 5 (MDA5). This results in growth inhibition and tumor inflammation, respectively, and profoundly sensitizes tumors to immunotherapy [42]. Finally, demethylation positively regulates the transcriptional activity of some immune-related genes, including PD-L1 and IFN signaling pathway genes, sensitizing it to anti-cytotoxic T-lymphocyte-associated protein-4 (CTLA-4) therapy [43]. 
In addition to the IFN- $\gamma$-related signaling pathway, alterations in other tumor genome, such as tumor oncogenes and suppressor genes pathways, and pathways related to tumor cell proliferation and infiltration, can also affect immunotherapy efficacy. Epidermal growth factor receptor (EGFR) and anaplastic lymphoma kinase (ALK) mutations have been shown to be associated with reduced response rates to ICIs and low TMB, and therefore the FDA does not recommend first-line ICIstreatment in patients with EGRF or ALK positive tumors $[44,45]$; certain types of mutations in MDM2/MDM4 and ARID1A can predict non-response to ICIs in high TMB tumors [46]; NSCLC with KRAS and STK11 comutated was associated with reduced response and shorter survival in three independent cohorts of patients treated with anti-PD-1 therapy [47], and STK11 deficiency was an independent indicator of poor anti-PD-1 response in NSCLC with KRAS mutant; however, at the 2020 American Association for Cancer Research (AACR) meeting, 33.7\% of patients in the Keynote-042 study (NCT02220894) update data were tested for STK11 and KEAP1, and the results showed that patients could benefit from pembrolizumab regardless of STK11 and KEAP1 status, but patients with STK11 mutations did not respond well to chemotherapy, but given that only $1 / 3$ of all patients had mutation detection, the results may be affected; in initial data from studies using targeted NGS panels suggested that duration of ICIs-treatment was associated with certain BRAF and MET alterations, but not TMB status [48]. NOTCH signaling pathway is associated with the occurrence, development and prognosis of tumors, especially with the biological function of cancer stem cells. Recent breakthrough findings have distinguished deleterious $\mathrm{NOTCH}$ mutation, showing that it can be used as a potential predictor of favorable ICI response in NSCLC, potentially via greater transcription of genes related to DNA damage response and immune activation [49]. Another tumor-specific inheritance that may influence ICIs efficacy is the aberrant expression of endogenous retroviruses (ERVs). Pan-cancer analysis identified a positive correlation of transcript expression of ERVs with T-cell activity in various tumors [50] and patient prognosis [51]. Furthermore, with the improvement of precision detection technology, the accurate analysis of negative mutation sites helps to identify the possibly effective ones. For example, the analysis of study data of second-line PD-1/L1 inhibitor therapy found that the mPFS of patients with KRAS G12C or G12V was significantly better than that of patients with KRAS mutations at other sites [52].

In addition, several pan-cancer biomarkers are recently approved by the FDA. For example, given the effective ORR of $35.5 \%$ and a disease control rate (DCR) of $82 \%$ in second-line cholangiocarcinoma patients treated with pemigatinib, a new targeted therapy, the recent FDA approval of pemigatinib for the treatment of previously treated patients with locally advanced or metastatic cholangiocarcinoma with fibroblast growth factor receptor 2 (FGFR2) fusion or rearrangement, and the comprehensive genomic analysis assay, FoundationOne CDx, developed by Foundation Medicine as a companion diagnostic. Also exciting is the recent FDA approval of the targeted anticancer drug capmatinib for the treatment of metastatic NSCLC with MET exon 14 skipping (METex14) mutations, including first-line patients and previously treated patients, also using FoundationOne $\mathrm{CDx}$ as a companion diagnostic to help detect specific mutations present in tumor tissue.

\section{Neoantigen load}

Neoantigen load, the number of mutations actually targeted by $\mathrm{T}$ cells, may be directly related to the response to ICIs [53-55]. A retrospective study showed that clonal neoantigen burden was associated with the longer OS in primary lung adenocarcinomas $(p=0.025)$ [53]. Traditionally, computational neoantigen predictions have focused on major histocompatibility complex (MHC) binding of peptides based on anchor residue identities, however, neoantigen loads identified by this method are generally not superior to overall TMB in predicting ICIs efficacy or survival [56]. In recent practice, this neoantigen can be assessed by the difference in predicted MHC-I binding affinity between the wild-type peptide and the corresponding mutant peptide, known as the differential agretopicity index (DAI), reflecting clinically relevant immunogenicity of tumor peptide [57]. A high DAI value indicates that the mutant peptide significantly increases binding affinity to MHC compared to the wild-type sequence and can generate more immune responses. Studies on previously published cohorts treated with three ICIs have shown that DAI outperforms TMB and the traditionally defined neoantigen load in predicting survival [58, 59]. In addition, low neoantigen intratumour heterogeneity might also be important for ICIs response. Analysis of the lung adenocarcinoma TCGA database found that combining high mutational load and low intratumoral neoantigen heterogeneity $(<1 \%)$ was significantly associated with OS and longer lasting clinical benefit than either variable alone [53]. Another reported method for assessing neoantigen foreignness is based on sequence homology of experimentally validated immunogenic microbial epitopes in the Immune Epitope Database (IEDB) [60], but it does not account for all possible human leukocyte antigen (HLA) contexts. In addition, the detection for neoantigen can be reflected from different levels such as peptides or genomes. A study developed the Neopepsee algorithm using a machine learning approach incorporating 
integration of nine immunogenicity features and gene mutation expression levels [61], and its application to melanoma and leukemia patients could improve the sensitivity and specificity of neoantigen prediction. Recently it has also been shown that promoter hypermethylation of neoantigen genes may be an important mechanism for immune editing and tumor immune evasion [62], indicating that combined detection of tumor genome and epigenetics may provide more information for immunotherapy efficacy.

\section{(ii). Tumor immune microenvironment phenotype biomarkers}

\section{PD-L1 expression}

Given that multiple studies in a variety of tumors have demonstrated a positive correlation between PD-L1 expression and response to ICIs or OS, even in first-line combination therapy [63-65], pembrolizumab is currently approved by the FDA for use in patients with PD$\mathrm{L}^{+}$(PD-L1 $\geq 50 \%$ of tumor cells in first-line treatment and $\geq 1 \%$ in second-line treatment) NSCLC and PD-L1 immunohistochemistry (IHC) as a companion diagnostic for anti-PD-1 therapy in NSCLC patients [66, 67]. However, some studies have not detected a significant correlation between PD-L1 expression and response to ICIs $[5,13,68]$, and PD-L1 negative patients can still benefit clinically with treatment with ICI or combination treatment with ICIs [69], with ORRs ranging from 11 to $20 \%$. Therefore, PD-L1 cannot yet be a comprehensive and independent biomarker in clinical practice in assessing efficacy, with following challenges still existing. Firstly, PD-L1 assay and antibody are not standardized [70]. Secondly, PD-L1 expression is temporally and spatially heterogeneous [71]. A study of 398 metastatic NSCLC treated with ICIs showed that PD-L1 varies substantially across different anatomic sites and during clinical course, being highest in adrenal, liver and lymph node metastases and lower in bone and brain metastases. And the predictive value of PD-L1 at different biopsy sites for the benefit of ICIs in NSCLC may vary: higher PD-L1 in lung or distant metastasis specimens was significantly associated with higher response rate, PFS and OS, while PD-L1 in lymph node metastasis biopsy was not associated with either response or survival [72]. Thirdly, positive score and cut-off value of PD-L1 expression is not standardized [71]. At present, PD-L1 positive score mainly focuses on the PD-L1 expression level of tumor cells, that is, tumor proportion score (TPS). But PD-L1 is also expressed on immune cells such as lymphocytes and macrophages and stromal cells, thus the investigators introduce the concept of combined positive score (CPS), which is the proportion score of the sum of PDL1 expressed by tumor cells and tumor-associated immune cells. In addition, PD-L1 expression on immune cells is also considered separately as one of the biomarkers to distinguish the benefit population, called immune positive score (IPS). Herbst et al. [73] showed that response to atezolizumab treatment was significantly associated with high levels of PD-L1 expression on the surface of TILs before treatment, but not with PD-L1 expression on tumor cells $(p=0.079)$. Finally, other inhibitory immune pathways may affect the response to ICIs therapy, including $\mathrm{T}$ cell immunoglobulin-3 (TIM-3), lymphocyte activation gene-3 (LAG-3), and V-domain Ig suppressor of T-cell activation (VISTA), which can be used as potential biomarkers for ICIs response.

\section{Biomarkers of tumor-infiltrating immune cells Overall immune status of tumor microenvironment}

The pattern of tumor immune infiltration can be broadly classified into immune-inflamed, immune-excluded and immune-desert [74]. Immune-inflamed is characterized by the presence of $\mathrm{CD}^{+}$and $\mathrm{CD} 4^{+} \mathrm{T}$ cells in the tumor parenchyma accompanied by the expression of immune checkpoint molecules [75], indicating a potential antitumor immune response to ICIs treatment [73]; immune-excluded is characterized by the presence of different immune cell types in the aggressive margin or stroma of tumor, but cannot infiltration into tumor parenchyma [74, 76]. Analysis of pre-treatment samples for anti-PD-1/PD-L1 revealed a relatively high abundance of $\mathrm{CD} 8^{+} \mathrm{T}$ cells at the invasive margin in responders, and serial sampling during treatment showed an increased infiltration of $\mathrm{CD}^{+} \mathrm{T}$ cells into tumor parenchyma [77]; while immune-desert phenotype is characterized by the absence of abundant $\mathrm{T}$ cells in the parenchyma or stroma of tumors and poor response to ICI-treatment [73]. Recently, immunoscore has been proposed as a valid marker for characterizing the immune status of tumor microenvironment (TME), classifying tumors, as well as predicting treatment response and prognosis [78], which involves the density of two lymphocyte populations $\left(\mathrm{CD}^{+}\right.$and memory $\left[\mathrm{CD} 45 \mathrm{RO}^{+}\right] \mathrm{T}$ cells) in the center and invading margin of tumor [79]. Mlecnik et al. [80] evaluated immunoscore in 599 specimens of stage I-IV colorectal tumor and confirmed that it was significantly associated with PFS, DFS, and OS, and multivariate analysis also showed the superiority of immunoscore in predicting disease recurrence and survival. The value of immunoscore to predicting ICIs efficacy is being validated internationally in clinical trials of melanoma and NSCLC [78].

A wider assessment of active immune responses within TME by immune gene expression profiling might effectively predict clinical benefit to ICIs strategies. Analysis of total RNA and genes that were substantially different between the patient groups in 50 pretreatment tumor biopsies revealed at least a 2.5 -fold increase in the 
expression of 22 immune-related genes in clinically active patients, including cytotoxic $\mathrm{T}$ cell markers (e.g., CD8A, perforin 1, granzyme B), Th1 cytokines or chemokines, MHC-II, and other immune-related genes (e.g., NKG7, IDO1) [81]. Ascierto et al. [82] screened more than 299 immune-related genes in patients with recurrent breast cancer 1-5 years after treatment and those without recurrence more than 7 years later, and found that five genes (IGK, GBP1, STAT1, IGLL5, and OCLN) were highly overexpressed in patients with recurrencefree survival. In addition, IFN- $\gamma$-induced immune gene signatures may be effective biomarkers for predicting the clinical benefit of treatment with ICIs. The study developed IFN- $\gamma$ scores combining multiple immune variables based on 10 gene signatures, which were then extended to 28 gene signatures in a validation set of 62 melanoma patients, including genes encoding IFN $-\gamma$, granzymes A/ B, perforin 1, IDO1, and other immune-related genes. Both gene scores showed significant associations with best overall response rate and PFS. Optimized cut-off values for IFN- $\gamma$ scores based on receiver operating characteristic curve (ROC curve) can achieve a positive predictive value of $59 \%$ for responders and a negative predictive value of $90 \%$ for non-responders [83].

\section{Immune cells with specific phenotypes in TME}

The phenotype of TILs also influences the efficacy of ICIs. The study used single-cell mRNA sequencing (scRNA-seq) data analysis to identify two major $\mathrm{CD}^{+} \mathrm{T}$ cell phenotypes within melanoma: memory-like and exhausted [84], the proportion of which is strongly correlated with response to ICIs. The research further found that the transcription factor 7 (TCF7) is selectively expressed in memory-like $\mathrm{T}$ cells, so the ratio of $\mathrm{CD}^{+} \mathrm{TCF}^{+}$to $\mathrm{CD} 8{ }^{+} \mathrm{TCF} 7-\mathrm{TILs}$ is strongly correlated with improved response and survival in melanoma patients treated with anti-PD-1 [84]. Balatoni et al. [85] found that 7 of 11 immune cells in TME were positively associated with $\mathrm{OS}$ after treatment, including $\mathrm{CD}_{4}^{+}$and $\mathrm{CD}^{+}{ }^{+} \mathrm{T}$ cells, $\mathrm{FOXP3}^{+} \mathrm{T}$ cells, $\mathrm{CD} 20^{+} \mathrm{B}$ cells, CD134 ${ }^{+}$ and $\mathrm{CD} 137^{+}$cells, and $\mathrm{NKp} 46^{+}$cells, and different immune cells at different sites were differently associated with clinical outcomes. Researchers found that only a small proportion of $\mathrm{CD}^{+}$TILs in tumors could recognize tumor mutation-associated antigens, while another population (bystander cells) was insensitive, and differential CD39 expression was the key molecule that distinguished the two populations [86]. Analysis of peripheral blood from a patient with colorectal cancer who responded rapidly to pembrolizumab treatment showed high expression of CD39 on CD8 ${ }^{+}$TILs, indicating that $\mathrm{CD} 39^{+} \mathrm{CD} 8^{+} \mathrm{TIL}$ may be a promising predictive biomarker [86]. The fact of very low level of CD39 expression on $\mathrm{CD}^{+}$TILs in $50 \%$ of EGFR-mutant NSCLC is consistent with their low response rate to anti-PD-1 immunotherapy.

In addition, a study showed that Fc domain glycan of the drug and $\mathrm{Fc} \gamma$ receptor $(\mathrm{Fc} \gamma \mathrm{R})$ expressed by the host bone marrow cells could determine the ability of PD-1tumor-associated macrophages (TAMs) to capture antiPD-1 drugs from the surface of $\mathrm{T}$ cells, which leads to PD-1 inhibitor resistance [87], and the association of TAMs and poor anti-PD-1 response was reported in melanoma cohorts [88]; anti-PD-1 response was associated with an increase in $\mathrm{CD}^{+} \mathrm{T}$ cells and natural killer cells (NK cells) and a decrease in macrophages [16]; and high intratumoral myeloid markers were associated with a nearly 6-fold decrease in mPFS after anti-PD-L1 therapy in RCC, emphasizing the inhibitory role of myeloid cells in response to ICIs [89]. In conclusion, immune cells in TME show a great promise in the development of predictive biomarkers for ICIs.

\section{Diversity of immune repertoires in TME}

Effective $T$ cell responses involve the activation and expansion of specific antigen-reactive $\mathrm{T}$ cell clones, so diversity of immune repertoire in intratumoral or peripheral may correlate with ICIs responses and can be quantified as richness and clonality [16]. However, the results seem to be complex, with some studies finding a positive correlation between TIL clonality and the response to ICIs before [90] or after [91] treatment, while others showing that only an increase in TIL clonality during treatment is associated with the response to anti$\mathrm{PD}-1[16,92]$; others show that intratumoral $\mathrm{T}$ cell clonality is not associated with survival, while peripheral $\mathrm{T}$ cell clonality is inversely associated with PFS and OS [93]. Tumeh et al. [77] further investigated whether baseline TILs have a narrow T cell receptor (TCR) repertoire, focusing on tumor-specific immune responses and whether this narrow TCR repertoire correlates with pembrolizumab responses. They found that responding patient had more restricted usage of the TCR beta chain (ie, a more clonal, less diverse population) than patients with progressive disease, and showed a 10-times increase in these clones after treatment, implying a tumorspecific response to treatment in these patients. Notably, baseline TCR clonality was not highly correlated with TIL density, suggesting that some patients with restricted TCR clonality specific for tumor antigens may still benefit from anti-PD-1 therapy even though TIL density is low. Recently, researchers have proposed the immune repertoire (IR)-Index, the average frequency of shared TCR clones in T clones in TILs and peripheral $\mathrm{PD}-1^{+} \mathrm{CD}^{+} \mathrm{T}$ cells. They found that neoantigenstimulated TCR agreed with IR-Index, and patients with high IR-index had better immune activation and higher gene expression profiles (GEPs) score, subsequently they 
confirmed the predictive value of IR-index to ICIs efficacy (DCR/PFS). But considering that it is difficult to sort out $\mathrm{PD}-1^{+} \mathrm{CD} 8^{+} \mathrm{T}$ cells in tumor tissue, based on two separate patient cohorts, a research confirmed that TCR repertoire diversity and clonality of peripheral PD$1^{+} \mathrm{CD} 8^{+} \mathrm{T}$ cells may serve as noninvasive predictors of clinical outcomes after ICIs in patients with NSCLC [94]. The viewpoints of T cell diversity and TCR clonality as markers of ICIs efficacy need to be further validated in a large patient population.

\section{(iii)Liquid biopsy biomarkers}

\section{Peripheral blood cell biomarkers}

Peripheral blood is a non-invasive source to explore potential biomarkers for ICIs, and although associations with clinical benefit and survival have been observed, its effectiveness has not been validated in prospective studies. Analysis of melanoma treated with ipilimumab showed that improved OS and PFS were associated with baseline values of peripheral blood components, including low absolute neutrophil count, low neutrophil-tolymphocyte ratio (NLR), low absolute monocyte count, low frequency of myelogenous suppressor cells, high frequency of FoxP3 $3^{+}$Treg cells, high lymphocyte frequency, high eosinophil count; and clinical benefit also associated with the dynamic changes of blood markers during treatment, including decreased $\mathrm{FoxP}^{+}$Treg concentrations and increased lymphocyte and eosinophil counts [95]. Reports in patients with melanoma treated with pembrolizumab and in patients with NSCLC treated with nivolumab have shown that NLR is associated with worse tumor response [96, 97]. Multivariate analysis in melanoma patients treated with anti-PD-1 antibodies showed that NLR was the only factor associated with worse ORR and shorter PFS, indicating that NLR is a strong predictor of worse outcome in patients treated with ICI [96]. Low baseline lactate dehydrogenase (LDH) levels, high relative/absolute eosinophil counts, and relative lymphocyte counts were associated with prolonged OS in anti-PD-1 and CTLA-4 treated melanoma [97, 98]. Given that previous studies have proposed the importance of baseline derived NLR (dNLR) and LDH levels as prognostic markers, a recent study proposed a composite prognostic index that comprehensively takes the two factors into account, lung immune prognostic index (LIPI), which characterized 3 risk groups: good, intermediate, and poor [99]. The analysis of 3987 patients with advanced NSCLC in 11 randomized trials showed that patients with good LIPI score who received ICI were associated with significantly better PFS and OS compared with patients with poor LIPI score, which was not observed in patients received chemotherapy [99].
The study of melanoma treated with ipilimumab showed that the percentage of baseline $\mathrm{CD}_{4} 5 \mathrm{RO}^{+}$/ $\mathrm{CD}^{+} \mathrm{T}$ cells was $\leq 25 \%$ in $80 \%$ of non-responders and $\geq$ $30 \%$ in all responders $(p<0.01)$ [100]. CyTOF analysis of melanoma treated with ICIs showed that the abundance of $\mathrm{CD}^{+} 9^{+}$and $\mathrm{MIP}^{+} \beta^{+}$NK cells [101] and CD $14^{+}$CD $16^{-}$HLA-DR ${ }^{\text {hi }}$ cells [102] were predictive biomarkers of response to anti-PD-1 therapy. In addition, ipilimumab treatment of melanoma with baseline high levels of circulating Tregs was associated with OS, possibly as a target for ipilimumab antibody ADCC due to its high CTLA-4 expression; whereas decreased or stabilized circulating Tregs at 12 weeks since ipilimumab initial administration was significantly associated with better DCR and OS [98]. Inducible T cell co-stimulator (ICOS) is costimulatory molecule expressed by activated $\mathrm{T}$ cells and Tregs. Analysis of surgical tissues and peripheral blood before and after treatment showed that anti-CTLA-4 treatment could induce ICOS pathway activation, and $\mathrm{CD}^{+}{ }^{+} \mathrm{ICOS}^{+} \mathrm{T}$ cells could produce IFN- $\gamma$ and recognize tumor antigens [103]. In addition, a recent report correlated the detection of circulating tumor cells (CTCs) in peripheral blood with the metastatic process of tumors, and PD-L1 is highly expressed in CTCs from patients with advanced head and neck cancer, suggesting that $\mathrm{PD}-\mathrm{L} 1^{+} \mathrm{CTC}$ may be a predictive biomarker of response to ICIs [104].

\section{Biomarkers of circulating tumor DNA}

The detection of circulating tumor DNA (ctDNA) can obtain tumor genomic information related to the response to ICIs, although the sensitivity or specificity has yet to be improved. Multiple studies showed that high mutation number of ctDNA was associated with improved OS and poor prognosis in patients with different cancer types treated with ICIs [105, 106]; Lee et al. [107] demonstrated that melanoma patients with persistently elevated ctDNA during PD-1 antibody therapy showed worse response and shorter PFS and OS. In addition, ctDNA can be a useful marker for identifying pseudoprogression during ICIs treatment. 9 patients with melanoma appeared pseudoprogression after ICIs therapy were reported to have favorable ctDNA profiles (defined as undetectable ctDNA or detectable ctDNA at baseline followed by $>10$-times decrease in ctDNA), while 18 of 20 patients with true progression had unfavorable ctDNA profiles [108]. The association of bTMB level based on ctDNA and clinical benefit with anti-PD-1/L1 therapy was validated in tumor patients, confirming that it is a promising predictive biomarker. NCC-GP150 established using optimized gene panel size and algorithms is feasible for bTMB evaluation and bTMB can be used as a biomarker of clinical benefit in NSCLC patients treated with ICIs [109]. Another similar study 
showed that $\mathrm{tTMB}$ are strongly correlated (Spearman 0.6, Pearson 0.7) with bTMB evaluated with a 500-gene panel, which may serve as a potential biomarker for the efficacy of single and dual immunotherapy when cut-off value at $20 \mathrm{mt} / \mathrm{Mb}$ [110]. In addition, dynamic monitoring of ctDNA can provide $\triangle \mathrm{bTMB}$ information to predict the responsiveness in the treatment process in a non-invasive manner, potentially improving the sensitivity and specificity of response prediction.

\section{Other circulating molecular biomarkers}

Exosomes in the plasma can also provide information about the tumor and immunotherapy. Lower baseline levels and increases during treatment in circulating exosomal PD-L1 in melanoma patients were associated with response to pembrolizumab [111]. However, in another study of melanoma or NSCLS treated with anti-PD-1, the expression of PD-1 mRNA in the exosomes was higher at baseline and significantly decreased after treatment in patients with response, while it was stable in patients with stable disease and increased in patients with progressive disease after treatment [112]. Therefore, protein and transcripts of exosomal PD-L1 may provide contradictory information on the response to ICIs and require large-scale prospective studies for validation. In addition, RNA sequencing analysis of PD-L1 inhibitorresistant NSCLC patients revealed the presence of PDL1 variant fragments (v242 and v229, which retain the PD-1 binding domain) in vivo and in peripheral blood and pleural effusion, resistant patients with variant had much higher sPD-L1 concentrations. Experiments in vitro and vivo have confirmed the inhibitory effect of PD-L1 variant fragments on $\mathrm{T}$ cell activity [113], indicating a poor efficacy response.

In addition, other potential predictive biomarkers for ICIs efficacy have been preliminarily explored [114], including soluble proteins (e.g., sCD163, sNKG2DLs), cytokines and inflammatory factors [e.g., tumor necrosis factor (TNF)- $\alpha$, interleukin (IL)-6, C-reactive protein (CRP)]. Baseline serum LDH is often an independent factor for poor prognosis and shorter OS with ipilimumab or pembrolizumab in patients with advanced melanoma $[97,115]$. Several studies showed that in patients with various cancers treated with ICIs, high baseline LDH was associated with poor anti-tumor response $[116,117]$. Weber et al. [118] analyzed baseline levels of CRP and IL-6 in serum from patients with melanoma who participated in 3 different clinical trials and levels above baseline median were found to be significantly associated with poor response and shorter survival after nivolumab treatment, and similar results were found with ipilimumab and combination therapy. In vitro studies revealed that purified CRP significantly inhibited $\mathrm{T}$ cell activation and proliferation at concentrations >
$10 \mu \mathrm{g} / \mathrm{mL}$ [118]. Studies have also demonstrated that IL6 has an immunosuppressive effect under certain conditions, including induction of myeloid-derived suppressor cells (MDSCs), which may explain the above phenomenon [119]. Besides, two retrospective studies involving approximately 3000 patients found that high baseline levels of plasma IL-8 were significantly associated with poor prognosis with PD-1/L1 inhibitors therapy and may be a driver of resistance to ICIs [120, 121]. scRNA-seq of the immune compartment showed that IL-8 is primarily expressed in circulating and intratumoral myeloid cells, and had an inhibitory effect on adaptive immunity. High IL-8 levels were associated with higher tumor neutrophil/monocyte infiltration, poorer antitumor activity of effector $\mathrm{T}$ cells, as well as weaker antigen presentation. Patients with both a higher $\mathrm{T}$ cell effect profile score and lower plasma IL-8 levels can obtain the greatest benefit from ICIs therapy.

\section{(iv).Host-related markers}

\section{General characteristics}

Studies have shown that gender differences are associated with the responsiveness to anti-tumor immune. A meta-analysis including 20 randomized controlled trials (RCTs) of ICIs $(n=11,351)$ reported that gender difference in the efficacy ICIs was significant $(p=0.0019)$, with pooled OS hazard ratio being $0.72(95 \%$ CI $0.65-$ $0.79)$ in male patients and $0.86(95 \% \mathrm{CI} 0.79-0.93)$ in female patients [122]. In another meta-analysis of a large number of melanoma and NSCLC patients treated with ICIs, both PFS and OS were significantly longer in male patients than in female patients, and this difference was more pronounced in melanoma patients and antiCTLA-4 antibodies [123]. Aging is associated with restricted immune function with significant effects on both innate and adaptive immune responses [124]. A preclinical study showed that aged mice had significantly increased tumor responses to anti-PD-1 agents compared with young mice, considered to be associated with a lower proportion of Tregs in aged mice [125]. Consistently, melanoma patients over 60 years old have a significantly higher tumor response to pembrolizumab than patients under 60 , and the likelihood of response increases with age [125]. However, different results have also been reported by Nishijima et al. with an association between age less than 75 years and better ORR in patients treated with ICIs [126]. The Checkmate-171 trial showed that patients $\geq 70$ years of age had comparable tolerability and efficacy to the overall population [127]. However, at present, the inclusion and representativeness of the elderly in clinical studies are still insufficient.

Besides, studies of the effect of performance status (PS) on the efficacy of ICIs have shown that good PS are associated with lower tumor burden and a predominance of 
immune cell function in TME. In the Checkmate-171, patients with PS $=2$ had inferior efficacy to the overall population [127], and real-world data in Israel again suggested that patients with PS $\geq 2$ had inferior efficacy to the overall population [128]. A study reported by ASCO in 2018 (Justin F. et.al. 2018 ASCO abstract\#9011) showed that the TMB of smoking patients with NSCLC in the two groups with equivalent PD-L1 expression was higher, and PFS and duration of response (DOR) in smoking patients with high PD-L1 expression (TPS $\geq 50 \%$ ) were longer. Differences in body fat distribution also affects tumor prognosis and immunotherapy [129]. A study showed obesity induces $\mathrm{T}$ cell exhaustion and dysfunction by affecting PD-1 expression through STAT3 signaling, leading to increased immune aging and promoting tumor growth and progression [130]. However, targeting receptors on activated $\mathrm{T}$ cells or chimeric antigen receptor (CAR)- $\mathrm{T}$ cells in immunotherapy may help enhance $\mathrm{T}$ cell function, especially in the presence of high leptin. Studies have found that obese mice also showed a significantly better response to anti-PD-1 without significant toxic side effects [130], which was reproduced in multiple cancer populations receiving ICIs [131], with higher body mass index (BMI) $(\mathrm{BMI}>30)$ patients showing reduction in tumor burden and improvement in PFS and OS. Although the mechanisms by which baseline general characteristics influence the efficacy of ICIs have not been fully demonstrated, they can be used as stratification factors for efficacy and tumor prognosis in future trials to gradually expand the understanding.

\section{Intestinal commensal microbiota}

The commensal microbiota plays a key role in the immune response, with gut bacteria significantly associated with improved responses to ICIs in humans [132]. Four independent studies analyzing baseline fecal samples found that different specific intestinal bacterium were associated with response to ICIs in melanoma [133-135], NSCLC, RCC, and urothelial carcinoma [132]; Sivan et al. [136] reported that commensal bifidobacteria enhanced anti-PD-1 antibody response by enhancing DC function in mice; PFS and OS after ipilimumab treatment in melanoma patients with baseline microbiota enriched Faecalibacterium species and other Firmicutes were better than those with baseline microbiota enriched Bacteroides [134]; in addition, Routy et al. [132] revealed a correlation between clinical response and the relative abundance of Akkermansia muciniphilia, which enhanced the efficacy of PD-1 antibodies in an IL-12-dependent manner. The impact on the efficacy of ICIs may be related to different cancer types, microbial sequencing and analysis techniques, geographical distribution of intestinal bacteria, as well as antibiotic treatment.

\section{Host germline genetics}

HLA genes are the most polymorphic genes in the human genome and encode key components of immunogenicity. HLA-I diversity is characterized by significant sequence variation in peptide-binding regions, termed the human immunopeptidome [137]. Analysis of 1535 patients with ICI-treated tumors found that the presence of a more diverse array of HLA-I molecules was associated with increased survival [138], possibly due to its broader presentation of tumor antigens $[139,140]$. Patients who are heterozygous for all HLA-I loci in patients receiving anti-PD-1 therapy have a higher ontreatment clonal expansion of TCR repertoire than homozygous patients [139]. A study showed that HLA loss of heterozygosity (HLA LOH) occurs in $45.1 \%$ of all patients with advanced disease and varies by tumor type [14]. The concordance of HLA LOH detected by WES and multi-genic panels is high and suggests that HLA $\mathrm{LOH}$ may be associated with immune escape, resulting in the resistance to immunotherapy [141]. In addition, specific HLA-I supertypes, such as the HLAB44 supertype allele, are associated with improved survival in melanoma patients treated with ICIs [139]. Other host immune-related gene polymorphisms, including HLA-II genes, non-classical HLA-i genes, NF-KB, and JAKSTAT family members, have also been shown to be associated with tumor response to ICIs [138]. Future studies are needed to further investigate the impact of host immune gene variation on ICIs efficacy.

\section{(v). Immune-related adverse events}

Since ICIs may cause tumor regression and immunerelated adverse events (irAEs) through enhanced immune responses, several studies have shown a relevance of the two. irAEs are associated with tumor regression in patients with metastatic RCC or melanoma treated with ipilimumab [142, 143]. And the early development of overall irAEs was associated with better ORR and PFS in NSCLC patients treated with nivolumab [144]. However, multivariate analysis by Judo [145] showed that only low-grade irAEs, but not high-grade irAEs, were associated with better response to anti-PD-1 blockade in patients with non-melanoma. In addition, different types of irAEs are associated with immunotherapeutic responses in different tumor types. Fujisawa et al. [146] demonstrated an association between endocrine irAEs and OS and better prognosis in melanoma patients treated with ipilimumab after nivolumab; likewise, thyroid dysfunction in NSCLC patients treated with anti-PD-1 was statistically associated with OS and PFS [147]. The development of vitiligo is associated with a better response to ipilimumab in melanoma patients [148], may representing a common immune response against 
antigens shared by melanocytes and melanomas. Several studies showed that other skin irAEs were also associated with better outcomes in cancer $[149,150]$. But the controversial finding reported that the occurrence of skin toxicities, except for vitiligo, were related to a shorter OS of melanoma patients treated with ipilimumab after nivolumab [146]. Since skin irAEs include various types of skin disorders, such as pruritus, psoriasis, and lichenoid toxicity, the association of each skin irAE with outcome may vary. However, given that ICIs may cause tumor regression and irAEs by enhancing immune responses, some biomarkers that have been explored to predict the occurrence of irAEs, such as T cell diversity, cytokines and inflammatory factors, different gut microbiome, may also be predictive biomarkers of ICIs-efficacy. Therefore, in clinical practice, how to better use biomarkers to achieve the best efficacy while experienced minimal toxicity is a difficult problem to explore in the future.

There provides an overview of predictive biomarkers for ICIs efficacy in Fig. 1, and details of some factors in Table 1.

\section{Exploration of predictive markers by ICl types}

In addition, considering that the type of ICIs is more correlated with treatment, it seems more reasonable to explore biomarkers that can predict the efficacy of different ICIs. Studies have shown an association between tumor autoantigen expression and improved ICIresponse. Eight-gene cluster known as the "anti-CTLA-4 resistance associated MAGE-A (CRMA)" cluster is associated with poor response to anti-CTLA-4 rather than anti-PD-1 therapy [151]. The exact mechanism is unknown, but may be related to the idea that the expression of CRMA leads to a reduction or defect in autophagy, which in turn interferes with antigen processing and presentation. Therefore, CRMA expression is considered to be a predictive biomarker for antiCTLA-4 therapy rather than a predictor of overall disease prognosis, and CRMA gene expression may be used to identify patients who respond to combination therapy of anti-CTLA-4 and anti-PD-1 [151]. The researchers analyzed the expression MHC-I and II protein in tumor cells from previously untreated patients with advanced melanoma, and correlated the results with transcriptomic and genomics analyses [152]. They found that MHC proteins showed different sensitivities to CTLA-4 and PD-1 blockers. Major (>50\%) or complete loss of MHC-I expression on membranes of melanoma cell was associated with transcriptional repression of HLA-A, HLA-B, HLA-C, and B2M in 78/181 patients (43\%), which could predict the resistance to anti-CTLA-4

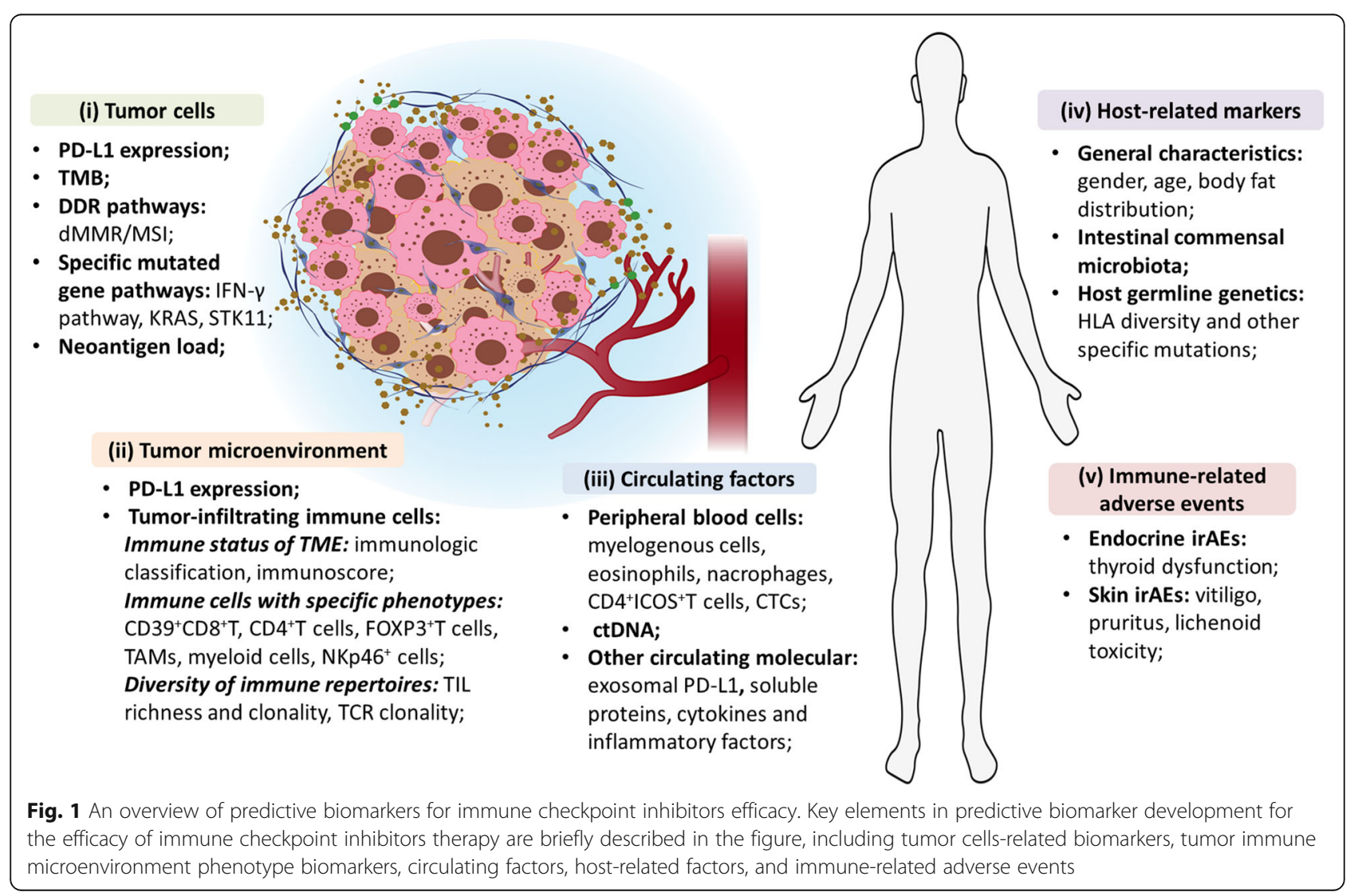


Table 1 Details of some factors that predict response to immune checkpoint inhibitor therapy

\begin{tabular}{|c|c|c|c|c|}
\hline Type of marker & Marker & $\begin{array}{l}\text { Association with } \\
\text { clinical outcome }\end{array}$ & Cancer type & $\begin{array}{l}\text { Tissue type for marker } \\
\text { assessment }\end{array}$ \\
\hline \multirow[t]{9}{*}{$\begin{array}{l}\text { Tumor genome and neoantigen } \\
\text { biomarkers }\end{array}$} & Tumor mutation burden & Positive or negative & $\begin{array}{l}\text { Multiple } \\
\text { tumor types }\end{array}$ & Tumor tissue or blood \\
\hline & PD-L1 expression in tumor & Positive & $\begin{array}{l}\text { Multiple } \\
\text { tumor types }\end{array}$ & Tumor tissue \\
\hline & lindel & Positive & $\begin{array}{l}\text { Multiple } \\
\text { tumor types }\end{array}$ & Tumor tissue \\
\hline & SCNAS & Positive & $\begin{array}{l}\text { Multiple } \\
\text { tumor types }\end{array}$ & Tumor tissue \\
\hline & DNAm, e.g., HOX gene methylation & Positive & NSCLC & Tumor tissue or blood \\
\hline & $\begin{array}{l}\text { DDR pathways, e.g., dMMR/MSI, BER, } \\
\text { HRR; }\end{array}$ & Positive & $\begin{array}{l}\text { Multiple } \\
\text { tumor types }\end{array}$ & Tumor tissue \\
\hline & $\begin{array}{l}\text { IFN- } \gamma \text { pathway genes, IFNGR1/2, JAK1/2, } \\
\text { and IRF1 }\end{array}$ & Negative & $\begin{array}{l}\text { Multiple } \\
\text { tumor types }\end{array}$ & Tumor tissue or blood \\
\hline & STK11 & Positive or Unknown & NSCLC & Tumor tissue or blood \\
\hline & $\begin{array}{l}\text { Neoantigen load, low neoantigen } \\
\text { intratumour heterogeneity }\end{array}$ & Positive & $\begin{array}{l}\text { Multiple } \\
\text { tumor type }\end{array}$ & Tumor tissue \\
\hline \multirow[t]{4}{*}{$\begin{array}{l}\text { Tumor immune microenvironment } \\
\text { phenotype biomarkers }\end{array}$} & PD-L1 expression in TME & Positive & $\begin{array}{l}\text { Multiple } \\
\text { tumor types }\end{array}$ & Tumor tissue \\
\hline & Immune-inflamed TME & Positive & $\begin{array}{l}\text { Multiple } \\
\text { tumor types }\end{array}$ & Tumor tissue \\
\hline & T cell repertoire clonality & Positive & $\begin{array}{l}\text { Multiple } \\
\text { tumor types }\end{array}$ & Tumor tissue or blood \\
\hline & $\mathrm{CD} 39+\mathrm{CD} 8+\mathrm{TIL}$ & Positive & NSCLC, RCC & Tumor tissue or blood \\
\hline \multirow[t]{6}{*}{ Liquid biopsy biomarkers } & NLR & Negative & $\begin{array}{l}\text { Melanoma, } \\
\text { NSCLC }\end{array}$ & Blood \\
\hline & $\begin{array}{l}\text { High mutation number of ctDNA or } \\
\text { favorable ctDNA profiles }\end{array}$ & Positive & $\begin{array}{l}\text { Multiple } \\
\text { tumor types }\end{array}$ & Blood \\
\hline & LDH & Negative & Melanoma & Blood \\
\hline & IL-8 & Negative & $\begin{array}{l}\text { Multiple } \\
\text { tumor types }\end{array}$ & Blood \\
\hline & Exosomal PD-L1 & Positive or negative & $\begin{array}{l}\text { Melanoma, } \\
\text { NSCLC }\end{array}$ & Blood \\
\hline & PD-L1 variant fragments & Negative & NSCLC & Blood \\
\hline \multirow[t]{6}{*}{ Host-related markers } & Gender & Male: positive & $\begin{array}{l}\text { Multiple } \\
\text { tumor types }\end{array}$ & - \\
\hline & Age & $\begin{array}{l}\text { Positive or negative or } \\
\text { Unknown }\end{array}$ & $\begin{array}{l}\text { Multiple } \\
\text { tumor types }\end{array}$ & - \\
\hline & Body fat distribution & Positive & $\begin{array}{l}\text { Multiple } \\
\text { tumor types }\end{array}$ & - \\
\hline & Specific Intestinal microbiota & Positive or negative & $\begin{array}{l}\text { Multiple } \\
\text { tumor types }\end{array}$ & Oral or gut \\
\hline & HLA-I diversity & Positive & $\begin{array}{l}\text { Melanoma, } \\
\text { NSCLC }\end{array}$ & Blood \\
\hline & $\mathrm{HLA} L O H$ & Negative & Melanoma & Tumor tissue \\
\hline irAEs & irAEs in different organs & Positive or Unknown & $\begin{array}{l}\text { Multiple } \\
\text { tumor types }\end{array}$ & - \\
\hline
\end{tabular}

$P D$ - L1 programmed cell death-ligand 1, RCC renal cell carcinoma, NSCLC non-small cell lung cancer, $T M B$ tumor mutation burden, indel insertion and deletion, SCNAs somatic copy number alterations, MMR mismatch repair, $A M M R$ MMR deficiency, MSI microsatellite instability, TLL tumor infiltrating lymphocyte, POLE/POLD1 polymerase gene epsilon/delta 1, BER base excision repair, HRR homologous recombination repair, DDR DNA damage response, $H L A$ human leukocyte antigen, TME tumor microenvironment, NLR neutrophil-to-lymphocyte ratio, ctDNA circulating tumor DNA, IL-8 interleukin-8, LDH lactate dehydrogenase, irAE immunerelated adverse event, DNAm DNA methylation HLA LOH HLA loss of heterozygosity 
antibody therapy but not anti-PD-1 therapy. MHC-II expression was observed in $>1 \%$ of melanoma cells in $55 /$ 181 (30\%) patients, and correlated with IFN- $\gamma$ and its mediated gene signature, which could predict the response to anti-PD-1 but not anti-CTLA-4 therapy [152]. Thus, MHC-I expression is required for the primary response against CTLA-4 for melanoma, while the primary response to anti-PD-1 is associated with pre-existing IFN- $\gamma$-mediated immune activation. Therefore, the exploration of markers to predict the therapeutic efficacy or resistance of different ICIs is also essential. More studies are expected in the future to analyze the mechanisms of action of different ICIs and their interactions with tumors in depth.

\section{Comprehensive predictors of ICls efficacy}

The current understanding of the clinical response to ICIs-treatment suggests that any single biomarker cannot effectively identify the benefit populations. The specificity and efficacy of prediction will be greatly improved when combination of multiple factors is used as a composite variable to capture immune status. Rizvi et al. [153] found that TMB and PD-L1 were two independent factors affecting the efficacy of immunotherapy, while patients with both high levels of TMB and positive PD-L1 had the highest duration of benefit rate; another study showed that NSCLC patients with both high TIL density and high PD-L1 expression treated with PD-L1 inhibitor had the highest positive predictive value of ORR and the longest PFS [154]; and Yu et al. [155] further demonstrated that the comprehensive variables of three predictive markers, CD8 ${ }^{+} \mathrm{TIL}, \mathrm{PD}-\mathrm{L} 1$ expression, and TMB, were associated with improved OS and PFS compared with a single biomarker or two of the three biomarkers. Furthermore, the use of big data analysis to predict markers of immunotherapy efficacy helps to establish a new framework for precise treatment of tumors. A study of 4 groups of clinical trials covering 22 cancer types and more than 300 patients evaluated the relationship between biomarkers and best overall responses (BOR), PFS. It was found that TMB, T cellinflamed GEPs were associated with the efficacy of clinical immunotherapy, and the higher TMB, the higher ORR [156].

In addition, developing predictive models by integrating different types of data based on different components of tumor-host interactions seems to have a good prospect. A research team created two neoantigen immune fitness models by computational biology methods, namely, the neoantigen quantity model, mainly statistically analyzing the number of tumor antigens, and the neoantigen quality fitness model, involving various factors such as the similarity between tumor antigens and pathogen antigens and the binding ability to TCR [157].
The results showed that only the neoantigen quality fitness model could better predict the postoperative survival of patients with pancreatic cancer. Another study developed a new neoantigen fitness model including three elements (tumor clonality, DAI, and microbial epitope homology), which was quantified as a nonlinear function of alignment scores, and the results showed that the model incorporating all three elements successfully predicted survival in all three ICI-treatment cohorts [60]. But before applying the model more broadly, it is necessary to identify unique parameters for each cancer species and/or therapeutic agent [158]. Jiang et al. [159] designed a completely new computational architecture, TIDE score ratio biomarkers (tumor mutation load, PDL1 level, and INF- $\gamma$ ), namely tumor immune dysfunction and rejection scores. It reveals the impact of tumor infiltration levels of different immune cell types on overall survival of patient by analyzing the TCGA and PRECOG databases and synthesizing different types of tumor immune escape mechanisms. Using this framework and pretreatment RNA-Seq or NanoString tumor expression profiling, they have identified that TIDE more accurately predicts the outcome of melanoma patients treated with first-line anti-PD-1 or anti-CTLA-4 than other biomarkers such as PD-L1 levels and TMB. TIDE also revealed novel candidate regulators of resistance to ICIs, such as SERPINB9, demonstrating utility for immunotherapeutic studies.

The combined detection of independent predictive markers makes more patients to receive ICIs and expands the beneficiary population, while for interacting markers, a bioinformatics-based predictive-model can be established according to different impact weights of each factor and improve the accuracy of screening the beneficiary population by comprehensive consideration, and how to better utilize the interrelationship network of various markers is an aspect to be considered of comprehensive predictive-models; in addition, it should be explored how the combined prediction with multiple factors achieve the optimal cost-effectiveness to serve the clinical immunotherapy of tumors more effectively. In the future, it may be promising to obtain the most effectively comprehensive predictive-markers by extracting features with large samples and multiple dimensions and constructing multivariate models using machine learning and artificial intelligence.

\section{Summary and outlook}

In this review, we deeply analyze the exploration course and research progress of predictive biomarkers as an adjunctive tool to tumor immunotherapy in identifying ICIs efficacy. In recent years, predictive markers of ICIs efficacy have been gradually explored from the expression of intermolecular interactions within tumor cells to 
the expression of various molecules and cells in TME, even been extended to the exploration of circulating and host systemic markers, and gradually realized the process from the identification of single marker to the development of multifactorial synergistic predictive markers. The exploration of predictive biomarkers of ICIs efficacy indicates a complex interaction between the regulation of the immune system network and tumors, reflecting more comprehensively the complexity and diversity of the effects of immunotherapy on tumors and even the whole body. Nonetheless, the findings of some biomarkers explored in the review are contradictory and the mechanisms of action are not well understood, which need to be confirmed by further large-scale prospective studies, but these breakthrough findings offer a great promise for biomarker strategies with more accurate positive and negative predictive values that can be used routinely in clinical practice to assist patients with different malignancies in ICIs-based therapy management, monitor disease development, and conquer tumor resistant to immunotherapy.

With the development of basic technology research such as multiplex IHC, high-throughput sequencing technology, and microarray technology, more and more potential markers can be widely screened widely on a genomic scale and a variety of proteins and cell populations can be quantified. However, several knowledge gaps still exist. First, for single marker, further cognition of PD-L1 and TMB should be enhanced, while continuing to promote consistency evaluation of detection methods; Gene mutations show great potential in the evaluation and monitoring of the whole course of immunotherapy and should be continuously explored; There are still many unknowns about the exploration of markers of the immune microenvironment and host microenvironment, which needs to be understood from a deeper molecular perspective. Secondly, in view of the various emerging biomarkers and the disadvantages of every single marker to varying degrees, strategies combining two or more approaches to capture immune status may be more effective as composite predictive biomarkers for ICIs efficacy. The advantages of each marker should be fully utilized to lay the foundation for the development of multifactorial predictive models. Balancing the relationship between the scientificity, accessibility, and simple operation of the clinical application of each predictive marker/model is a challenge to consider in clinical research. Thirdly, the exploration of more simple and feasible prediction means in clinical practice. For example, the potential of liquid biopsy such as ctDNA in the whole process of efficacy evaluation and monitoring of immunotherapy should be fully developed. Predicting the long-term survival of immunotherapy based on biomarkers in peripheral blood is a potential development direction. Furthermore, the use of machine deep learning and artificial intelligence to explore the mechanisms and markers of immunotherapy efficacy and drug resistance is changing from fantasy to reality, which can be used as the direction of future scientific research and clinical exploration. Multivariate predictive models need to extract data features with large samples and multiple dimensions using machine learning, and integrate different types of data based on different components of tumor-host interactions for comprehensive validation and evaluation, including polymorphism data such as intratumoral genomic and molecular characteristics, tumor immune microenvironment phenotype, peripheral blood biomarkers and host-related factors. Finally, given that multiple patterns of atypical response, such as pseudoprogression, occur during immunotherapy, which significantly affect patient treatment and overall survival, it is also essential for the exploration of predictive markers to these special response pattern. In the future, through the scientific study of the availability of multiple markers and the exploration of feasibility and reproducibility in clinical practice, standardized predictive biomarkers (models) for ICIs response would be established to maximize the benefit of patients from these transformative treatments, ultimately prompting the field to develop towards precision immuno-oncology.

\section{Abbreviations}

CAR: chimeric antigen receptor; BOR: best overall responses; ICl: Immune checkpoint inhibitor; PD-1/PD-L1: Programmed cell death-1/programmed cell death-ligand 1; RCC: Renal cell carcinoma; HNSCC: Head and neck squamous cell carcinoma; ORR: Objective response rate; NSCLC: Non-small cell lung cancer; TMB: Tumor mutation burden; SCLC: Small cell lung cancer; HPV: Human papilloma virus; NCCN: National Comprehensive Cancer

Network; OS: Overall survival; DCR: Disease control rate; DCB: Durable clinical benefit; PFS: Progression-free survival; CGP: Comprehensive genomic profiling; NGS: Next generation sequencing; AF: Allele frequency; CNV: Copy number variation; nsSNV: Nonsynonymous single nucleotide variant;

SCNAs: Somatic copy number alterations; indel: Insertion and deletion; TCGA: The Cancer Genome Atlas; MMR: Mismatch repair; dMMR: MMR deficiency; MSI: Microsatellite instability; TIL: Tumor infiltrating lymphocyte; IDO: Indoleamine 2,3-dioxygenase; POLE/POLD1: Polymerase gene epsilon/ delta 1; BER: Base excision repair; HRR: Homologous recombination repair; DDR: DNA damage response; dsRNA: Double-stranded RNA;

ADAR1: Adenosine deaminase acting on RNA; MDA5: Melanoma

differentiation-associated protein 5; CTLA-4: Cytotoxic T-lymphocyteassociated protein-4; EGFR: Epidermal growth factor receptor; ALK: Anaplastic lymphoma kinase; FDA: Food and Drug Administration; AACR: American Association for Cancer Research; ERV: Endogenous retroviruse; MHC: Major histocompatibility complex; DAl: Differential agretopicity index; IEDB: Immune Epitope Database; HLA: Human leukocyte antigen; IHC: Immunohistochemistry; TPS: Tumor proportion score; CPS: Combined positive score; IPS: Immune positive score; TIM-3: T cell immunoglobulin-3; LAG-3: Lymphocyte activation gene-3; VISTA: V-domain Ig suppressor of Tcell activation; TME: Tumor microenvironment; ROC curve: Receiver operating characteristic curve; scRNA-seq: Single-cell mRNA sequencing;

TCF7: Transcription factor 7; FCYR: Fc domain glycan of the drug and FCY receptor; TAM: Tumor-associated macrophage; NK cell: Natural killer cell; TCR: T cell receptor; IR: Immune repertoire; GEP: Gene expression profile; NLR: Neutrophil-to-lymphocyte ratio; ICOS: Inducible T Cell Co-stimulator; CTC: Circulating tumor cell; ctDNA: Circulating tumor DNA; MDSC: Myeloidderived suppressor cell; TNF: Tumor necrosis factor; IL: Interleukin; CRP: Creactive protein; $\mathrm{LDH}$ : Lactate dehydrogenase; RCT: Randomized controlled 
trial; PS: Performance status; ASCO: American Society of Clinical Oncology; DOR: Duration of response; BMI: Body mass index; irAE: immune-related adverse event; WES: whole exome sequencing; HLA LOH: HLA loss of heterozygosity; dNLR: derived NLR; LIPI: lung immune prognostic index; FGFR2: fibroblast growth factor receptor 2; METex14: MET exon 14 skipping; CRMA: CTLA-4 resistance associated MAGE-A

\section{Acknowledgements}

Not applicable.

\section{Authors' contributions}

$R L$ Bai carried out the primary literature search, drafted and revised the manuscript, and participated in discussions. Z Lv and DS Xu helped modify the manuscript. JW Cui carried out the design of the research and literature analysis, drafted and revised the manuscript, and participated in discussions. All authors read and approved the final manuscript.

\section{Funding}

This work was supported by the National Natural Science Foundation of China (Grant 81672275 and 81874052); Project of Jilin Provincial Department of Education (Grant JJKH20190020KJ); Project of Department of Science and Technology of Jilin Province (Grants 20180101009JC and 20190303146SF);

\section{Availability of data and materials}

Not applicable.

\section{Ethics approval and consent to participate}

Not applicable.

\section{Consent for publication}

Not applicable.

\section{Competing interests}

The authors declare that there are no conflicts of interest.

Received: 4 June 2020 Accepted: 29 July 2020

Published online: 26 August 2020

\section{References}

1. Gong J, Chehrazi-Raffle A, Reddi S, et al. Development of PD-1 and PD-L1 inhibitors as a form of cancer immunotherapy: a comprehensive review of registration trials and future considerations [J]. Immunother Cancer. 2018; 6(1):8.

2. Akinleye A, Rasool Z. Immune checkpoint inhibitors of PD-L1 as cancer therapeutics [J]. J Hematol Oncol. 2019;12(1):92.

3. Robert C, Long GV, Brady B, et al. Nivolumab in previously untreated melanoma without BRAF mutation [J]. N Engl J Med. 2015:372(4):320-30.

4. Garon EB, Rizvi NA, Hui R, et al. Pembrolizumab for the treatment of nonsmall-cell lung cancer [J]. N Engl J Med. 2015;372(21):2018-28.

5. Brahmer J, Reckamp KL, Baas P, et al. Nivolumab versus Docetaxel in advanced squamous-cell non-small-cell lung Cancer [J]. N Engl J Med. 2015; 373(2):123-35

6. Legrand FA, Gandara DR, Mariathasan S, et al. Association of high tissue TMB and atezolizumab efficacy across multiple tumor types [J]. J Clin Oncol. 2018;36(15_suppl):12000.

7. Rosenberg JE, Hoffman-Censits J, Powles T, et al. Atezolizumab in patients with locally advanced and metastatic urothelial carcinoma who have progressed following treatment with platinum-based chemotherapy: a single-arm, multicentre, phase 2 trial [J]. Lancet. 2016;387(10031):1909-20.

8. Hellmann MD, Callahan MK, Awad MM, et al. Tumor mutational burden and efficacy of Nivolumab Monotherapy and in combination with Ipilimumab in small-cell lung Cancer [J]. Cancer Cell. 2018;33(5):853-61.e4.

9. Hellmann MD, Nathanson T, Rizvi H, et al. Genomic features of response to combination immunotherapy in patients with advanced non-small-cell lung Cancer [J]. Cancer Cell. 2018;33(5):843-52.e4

10. Hellmann MD, Ciuleanu TE, Pluzanski A, et al. Nivolumab plus Ipilimumab in Lung Cancer with a High Tumor Mutational Burden [J]. N Engl J Med. 2018; 378(22):2093-104.

11. Singal G, Miller $P G$, Agarwala $V$, et al. Association of Patient Characteristics and Tumor Genomics with Clinical Outcomes among Patients with non-
Small Cell Lung Cancer Using a Clinicogenomic database [J]. Jama. 2019; 321(14):1391-9.

12. Goodman AM, Kato S, Bazhenova L, et al. Tumor mutational burden as an independent predictor of response to immunotherapy in diverse cancers [J]. Mol Cancer Ther. 2017;16(11):2598-608.

13. Hanna GJ, Lizotte $P$, Cavanaugh $M$, et al. Frameshift events predict anti-PD1/L1 response in head and neck cancer [J]. JCI Insight. 2018;3(4):e98811.

14. Yarchoan M, Hopkins A, Jaffee EM. Tumor mutational burden and response rate to PD-1 inhibition [J]. N Engl J Med. 2017;377(25):2500-1.

15. Miao D. Margolis CA. Genomic correlates of response to immune checkpoint therapies in clear cell renal cell carcinoma [J]. Science. 2018; 359(6377):801-6.

16. Riaz N, Havel JJ, Makarov V, et al. Tumor and Microenvironment Evolution during Immunotherapy with Nivolumab [J]. Cell. 2017;171(4):934-49.e16.

17. Fuchs C, ÖZgüRoĞLu M, Bang Y-J, et al. The association of molecular biomarkers with efficacy of pembrolizumab versus paclitaxel in patients with gastric cancer (GC) from KEYNOTE-061[J]. J Clin Oncol. 2020;38:4512.

18. Shitara K, ÖZgÜRoĞLu M, Bang Y-J, et al. The association of tissue tumor mutational burden (tTMB) using the Foundation Medicine genomic platform with efficacy of pembrolizumab versus paclitaxel in patients (pts) with gastric cancer (GC) from KEYNOTE-061[J]. J Clin Oncol. 2020;38:4537.

19. Li W, Matakidou A, Ghazoui Z, et al. Molecular biomarkers to identify patients (pts) who may benefit from durvalumab (D; anti-PD-L1) \pm tremelimumab (T;anti-CTLA-4) in recurrent/metastatic head and neck squamous cell carcinoma (R/M HNSCC) from HAWK and CONDOR studies[]]. J Clin Oncol. 2020;38:6548.

20. Li W, Wildsmith S, Ye J, et al. Plasma-based tumor mutational burden (bTMB) as predictor for survival in phase III EAGLE study: Durvalumab (D) \pm tremelimumab (T) versus chemotherapy (CT) in recurrent/metastatic head and neck squamous cell carcinoma (R/M HNSCC) after platinum failure[J]. J Clin Oncol. 2020;38:6511

21. Kawazoe A, Yamamoto N, Kotani D, et al. TAS-116, an oral HSP90 inhibitor, in combination with nivolumab in patients with colorectal cancer and other solid tumors: An open-label, dose-finding, and expansion phase lb trial (EPOC1704)[J]. J Clin Oncol. 2020;38:4044.

22. Kowanetz M, Zou W Shames D, et al. OA20.01 tumor mutation burden (TMB) is associated with improved efficacy of Atezolizumab in $1 \mathrm{~L}$ and $2 \mathrm{~L}+$ NSCLC patients [J]. J Thorac Oncol. 2017;12(1):S321-2.

23. Rosenberg JE, et al. Atezolizumab as first-line treatment in cisplatinineligible patients with locally advanced and metastatic urothelial carcinoma: a singlearm, multicentre, phase 2 trial. Lancet. 2017;389:67.

24. Samstein RM, Lee CH, Shoushtari AN. Tumor mutational load predicts survival after immunotherapy across multiple cancer types[J]. Nat Genet. 2019:51(2):202-6.

25. Johnson DB, Frampton GM, Rioth MJ, et al. Targeted next generation sequencing identifies markers of response to PD-1 blockade [J]. Cancer Immunol Res. 2016:4(11):959-67.

26. Ramalingam S, Hellmann M, Awad M, et al. Abstract CT078: Tumor mutational burden (TMB) as a biomarker for clinical benefit from dual immune checkpoint blockade with nivolumab (nivo) + ipilimumab (ipi) in first-line (1L) non-small cell lung cancer (NSCLC): identification of TMB cutoff from CheckMate 568[J]. Cancer Res. 2018;78:CT078.

27. Fang $W, M a ~ Y$, Yin JC, et al. Comprehensive genomic profiling identifies novel genetic predictors of response to anti-PD- $(L) 1$ therapies in Non-Small Cell Lung Cancer[J].Clin Cancer Res. 2019;25(16):5015-26.

28. Wang Z, Duan J, Wang G, et al. Allele frequency-adjusted blood-based tumor mutational burden as a predictor of overall survival for patients with NSCLC treated with PD-(L)1 inhibitors [J]. J Thorac Oncol. 2020;15(4):556-67.

29. Cai L, Bai H, Duan J, et al. Epigenetic alterations are associated with tumor mutation burden in non-small cell lung cancer [J]. J Immunother Cancer. 2019;7(1):198.

30. Turajlic $\mathrm{S}$, Litchfield $\mathrm{K}, \mathrm{Xu} \mathrm{H}$, et al. Insertion-and-deletion-derived tumourspecific neoantigens and the immunogenic phenotype: A pan-cancer analysis [J]. Lancet Oncol. 2017:18(8):1009-21.

31. Davoli $\mathrm{T}$, Uno H, Wooten EC, et al. Tumor aneuploidy correlates with markers of immune evasion and with reduced response to immunotherapy [J]. Science. 2017;355(6322):eaaf8399.

32. Taylor AM, Shih J, Ha G, et al. Genomic and Functional Approaches to Understanding Cancer Aneuploidy [J]. Cancer Cell. 2018;33(4):676-89.e3.

33. Zhang $Y$, Sun $Z$, Mao $X$, et al. Impact of mismatch-repair deficiency on the colorectal cancer immune microenvironment [J]. Oncotarget. 2017;8(49):85526-36. 
34. Overman MJ, Lonardi S, Wong KYM, et al. Durable clinical benefit with Nivolumab plus Ipilimumab in DNA mismatch repair-deficient/ microsatellite instability-high metastatic colorectal Cancer [J]. J Clin Oncol. 2018;36(8):773-9.

35. Wang F, Zhao Q, Wang YN, et al. Evaluation of POLE and POLD1 mutations as biomarkers for immunotherapy outcomes across multiple Cancer types [J]. JAMA Oncol. 2019;5(10):1504-6.

36. Wang Z, Zhao J, Wang $G$, et al. Comutations in DNA damage response pathways serve as potential biomarkers for immune checkpoint blockade [J]. Cancer Res. 2018;78(22):6486-96.

37. Possick JD. Pulmonary toxicities from checkpoint immunotherapy for malignancy [J]. Clin Chest Med. 2017;38(2):223-32.

38. Darnell J, Kerr I, Stark G. Jak-STAT pathways and transcriptional activation in response to IFNs and other extracellular signaling proteins [J]. Science. 1994; 264(5164):1415-21.

39. Wang X, Haswell JR, Roberts CWM. Molecular pathways: SWI/SNF (BAF) complexes are frequently mutated in cancer--mechanisms and potentia therapeutic insights [J]. Clin Cancer Res. 2014;20(1):21-7.

40. Miao D, Margolis CA, Gao W, et al. Genomic correlates of response to immune checkpoint therapies in clear cell renal cell carcinoma [J]. Science. 2018;359(6377):801-6.

41. Pan $D$, Kobayashi $A$, Jiang $P$, et al. A major chromatin regulator determines resistance of tumor cells to T cell-mediated killing [J]. Science (New York, N. Y.). 2018;359(6377):770-5

42. Ishizuka JJ, Manguso RT, Cheruiyot CK, et al. Loss of ADAR1 in tumours overcomes resistance to immune checkpoint blockade [J]. Nature. 2019; 565(7737):43-8.

43. De Velasco G, Je Y, Bosse D, et al. Comprehensive meta-analysis of key immune-related adverse events from CTLA-4 and PD-1/PD-L1 inhibitors in Cancer patients [J]. Cancer Immunol Res. 2017;5(4):312-8.

44. Chae YK, Davis AA, Raparia K, et al. Association of Tumor Mutational Burden With DNA Repair Mutations and Response to Anti-PD-1/PD-L1 Therapy in Non-Small-Cell Lung Cancer [J]. Clin Lung Cancer. 2019;20(2):88-96.e6.

45. Rizvi H, Sanchez-Vega F, La K, et al. Molecular determinants of response to anti-programmed cell death (PD)-1 and anti-programmed death-ligand (PD-L)-ligand 1 blockade in patients with non-small-cell lung Cancer profiled with targeted next-generation sequencing [J]. J Clin Oncol. 2018;36(7):633-41.

46. Klempner SJ, Fabrizio D, Bane S. Tumor Mutational Burden as a Predictive Biomarker for Response to Immune Checkpoint Inhibitors: A Review of Current Evidence [J]. Oncologist. 2020;25(1):e147-59.

47. Skoulidis F, Goldberg ME. STK11/LKB1 Mutations and PD-1 Inhibitor Resistance in KRAS-Mutant Lung Adenocarcinoma [J]. Cancer Discov. 2018; 8(7):822-35.

48. Ross JS, Goldberg ME, Albacker LA, et al. 1138PDImmune checkpoint inhibitor (ICPI) efficacy and resistance detected by comprehensive genomic profiling (CGP) in non-small cell lung cancer (NSCLC)[J]. Annals of Oncology. 2017;28:mdx376.004

49. Zhang $\mathrm{K}$, Hong $\mathrm{X}$, Song $Z$, et al. Identification of deleterious NOTCH mutation as novel predictor to efficacious immunotherapy in NSCLC [J]. 2020:26(14):3649-61.

50. Rooney MS, Shukla SA, Wu CJ, et al. Molecular and genetic properties of tumors associated with local immune cytolytic activity[J]. Cell. 2015;160(1-2): 48-61.

51. Smith CC, Beckermann KE, Bortone DS, et al. Endogenous retroviral signatures predict immunotherapy response in clear cell renal cell carcinoma [J]. J Clin Invest. 2018;128(11):4804-20.

52. Tamiya Y, Zenke Y, Matsumoto S, et al. Abstract 9589: Therapeutic impact of mutation subtypes and concomitant STK11 mutations in KRAS -mutated nonsmall cell lung cancer (NSCLC): A result of nationwide genomic screening project (LC-SCRUM-Japan)[J]. J Clin Oncol. 2020;38(15_suppl):9589.

53. McGranahan N, Furness AJ, Rosenthal R, et al. Clonal neoantigens elicit $T$ cell immunoreactivity and sensitivity to immune checkpoint blockade [J]. Science. 2016:351(6280):1463-9.

54. Jiang $T$, Shi $T$, Zhang $H$, et al. Tumor neoantigens: from basic research to clinical applications [J]. J Hematol Oncol. 2019;12(1):93.

55. Yi M, Qin S, Zhao W, et al. The role of neoantigen in immune checkpoint blockade therapy [J]. Exp Hematol Oncol. 2018;7:28.

56. Rizvi NA, Hellmann MD, Snyder A, et al. Cancer immunology. Mutational landscape determines sensitivity to PD-1 blockade in non-small cell lung cancer [J]. Science. 2015;348(6230):124-8.
57. Duan F, Duitama J, Al Seesi S, et al. Genomic and bioinformatic profiling of mutational neoepitopes reveals new rules to predict anticancer immunogenicity [J]. J Exp Med. 2014;211(11):2231-48.

58. Ghorani E, Rosenthal R, McGranahan N, et al. Differential binding affinity of mutated peptides for MHC class I is a predictor of survival in advanced lung cancer and melanoma [J]. Ann Oncol. 2018;29(1):271-9.

59. Rech AJ, Balli D, Mantero A, et al. Tumor immunity and survival as a function of alternative Neopeptides in human Cancer [J]. Cancer Immunol Res. 2018;6(3):276-87.

60. Łuksza M, Riaz N, Makarov $\mathrm{V}$, et al. A neoantigen fitness model predicts tumour response to checkpoint blockade immunotherapy [J]. Nature. 2017; 551(7681):517-20.

61. Kim S, Kim HS, Kim E, et al. Neopepsee: accurate genome-level prediction of neoantigens by harnessing sequence and amino acid immunogenicity information [J]. Ann Oncol. 2018;29(4):1030-6.

62. Yi M, Dong B, Chu Q, et al. Immune pressures drive the promoter hypermethylation of neoantigen genes [J]. Exp Hematol Oncol. 2019;8:32.

63. Wolchok JD, Chiarionsileni V, Gonzalez R, et al. Updated results from a phase III trial of nivolumab (NIVO) combined with ipilimumab (IPI) in treatmentnaive patients (pts) with advanced melanoma (MEL) (CheckMate 067)[J]. J Clin Oncol. 2016:34:9505.

64. Gandhi L, Rodríguez-Abreu D, Gadgeel S, et al. Pembrolizumab plus Chemotherapy in Metastatic Non-Small-Cell Lung Cancer [J]. N Engl J Med. 2018;378(22):2078-92

65. Rouquette I, Taranchon-Clermont E, Gilhodes J, et al. Immune biomarkers in thymic epithelial tumors: expression patterns, prognostic value and comparison of diagnostic tests for PD-L1[J]. Biomark Res. 2019;7:28.

66. Topalian SL, Taube JM, Anders RA, et al. Mechanism-driven biomarkers to guide immune checkpoint blockade in cancer therapy [J]. Nat Rev Cancer. 2016;16(5):275-87.

67. Gibney GT, Weiner LM, Atkins MB. Predictive biomarkers for checkpoint inhibitor-based immunotherapy [J]. Lancet Oncol. 2016;17(12):e542-51.

68. Carbone DP, Reck M, Paz-Ares L, et al. First-line Nivolumab in stage IV or recurrent non-small-cell lung Cancer [J]. N Engl J Med. 2017;376(25):2415-26.

69. Wu K, Yi M, Qin S, et al. The efficacy and safety of combination of PD-1 and CTLA-4 inhibitors: a meta-analysis [J]. Exp Hematol Oncol. 2019;8:26.

70. Sacher AG, Gandhi L. Biomarkers for the clinical use of PD-1/PD-L1 inhibitors in non-small-cell lung Cancer: a review [J]. JAMA Oncol. 2016;2(9):1217-22.

71. Nishino M, Ramaiya NH, Hatabu H, et al. Monitoring immune-checkpoint blockade: response evaluation and biomarker development [J]. Nat Rev Clin Oncol. 2017;14(11):655-68.

72. Hong L, Negrao MV, Dibaj SS, et al. Programmed Death Ligand 1 Heterogeneity and its Impact on Benefit from Immune Checkpoint Inhibitors in Non-Small-Cell Lung Cancer.[J]. Ann Oncol. 2019;30(8):1311-20.

73. Herbst RS, Soria JC, Kowanetz M, et al. Predictive correlates of response to the anti-PD-L1 antibody MPDL3280A in cancer patients [J]. Nature. 2014; 515(7528):563-7.

74. Chen DS, Mellman I. Elements of cancer immunity and the cancer-immune set point [J]. Nature. 2017:541(7637):321-30.

75. Llosa NJ, Cruise M, Tam A, et al. The vigorous immune microenvironment of microsatellite instable colon cancer is balanced by multiple counterinhibitory checkpoints [J]. Cancer Discov. 2015;5(1):43-51.

76. Joyce JA, Fearon DT. T cell exclusion, immune privilege, and the tumor microenvironment [J]. Science. 2015;348(6230):74-80.

77. Tumeh PC, Harview CL, Yearley JH, et al. PD-1 blockade induces responses by inhibiting adaptive immune resistance [J]. Nature. 2014;515(7528):568-71.

78. Galon J, Fox BA, Bifulco CB, et al. Immunoscore and Immunoprofiling in cancer: an update from the melanoma and immunotherapy bridge 2015[J]. J Transl Med. 2016;14:273.

79. Bindea G, Mlecnik B, Angell HK, et al. The immune landscape of human tumors: implications for cancer immunotherapy [J]. Oncoimmunology. 2014; 3(1):e27456

80. Mlecnik B, Tosolini M, Kirilovsky A, et al. Histopathologic-based prognostic factors of colorectal cancers are associated with the state of the local immune reaction [J]. J Clin Oncol. 2011;29(6):610-8.

81. Ji RR, Chasalow SD, Wang $L$, et al. An immune-active tumor microenvironment favors clinical response to ipilimumab [J]. Cancer Immunol Immunother. 2012;61(7):1019-31.

82. Ascierto $\mathrm{ML}$, Kmieciak M, Idowu MO, et al. A signature of immune function genes associated with recurrence-free survival in breast cancer patients [J]. Breast Cancer Res Treat. 2012;131(3):871-80. 
83. Ribas A, Robert C, Hodi FS, et al. Association of response to programmed death receptor 1 (PD-1) blockade with pembrolizumab (MK-3475) with an interferon-inflammatory immune gene signature [abstract][J]. Polym Int. 2015;33(2):141-7.

84. Sade-Feldman M, Yizhak K, Bjorgaard SL, et al. Defining T Cell States Associated with Response to Checkpoint Immunotherapy in Melanoma [J]. Cell. 2018;175(4):998-1013 e1020.

85. Balatoni T, Mohos A, Papp E, et al. Tumor-infiltrating immune cells as potential biomarkers predicting response to treatment and survival in patients with metastatic melanoma receiving ipilimumab therapy [J]. Cancer Immunol Immunother. 2018;67(1):141-51.

86. Simoni $Y$, Becht $E$, Fehlings $M$, et al. Bystander CD8(+) T cells are abundant and phenotypically distinct in human tumour infiltrates [J]. Nature. 2018; 557(7706):575-9.

87. Sean, Arlauckas, Christopher, et al. In vivo imaging reveals a tumorassociated macrophage-mediated resistance pathway in anti-PD-1 therapy [J]. Sci Transl Med. 2017;9(389):eaal3604.

88. Neubert NJ, Schmittnaegel M. T cell-induced CSF1 promotes melanoma resistance to PD1 blockade [J]. Sci Transl Med. 2018;10(436):eaan3311.

89. McDermott DF, Huseni MA, Atkins MB, et al. Clinical activity and molecular correlates of response to atezolizumab alone or in combination with bevacizumab versus sunitinib in renal cell carcinoma [J]. Nat Med. 2018; 24(6):749-57.

90. Roh W, Chen PL. Integrated molecular analysis of tumor biopsies on sequential CTLA-4 and PD-1 blockade reveals markers of response and resistance [J]. Sci Transl Med. 2017;9(379):eaah3560.

91. Forde PM, Chaft JE, Smith KN, et al. Neoadjuvant PD-1 blockade in Resectable. Lung Cancer [J]. N Engl J Med. 2018;378(21):1976-86.

92. Inoue H, Park JH, Kiyotani K, et al. Intratumoral expression levels of PD-L1, GZMA, and HLA-A along with oligoclonal T cell expansion associate with response to nivolumab in metastatic melanoma [J]. Oncoimmunology. 2016:5(9):e1204507.

93. Hopkins AC, Yarchoan M, Durham JN, et al. T cell receptor repertoire features associated with survival in immunotherapy-treated pancreatic ductal adenocarcinoma [J]. JCl Insight. 2018;3(13):e122092.

94. Han J, Duan J, Bai H, et al. TCR repertoire diversity of peripheral PD-1(+ CD8(+) T cells predicts clinical outcomes after immunotherapy in patients with non-small cell lung Cancer [J]. Cancer Immunol Res. 2020;8(1):146-54.

95. Delyon J, Mateus C, Lefeuvre D, et al. Experience in daily practice with ipilimumab for the treatment of patients with metastatic melanoma: an early increase in lymphocyte and eosinophil counts is associated with improved survival [J]. Ann Oncol. 2013;24(6):1697-703.

96. Bagley SJ, Kothari S, Aggarwal C, et al. Pretreatment neutrophil-tolymphocyte ratio as a marker of outcomes in nivolumab-treated patients with advanced non-small-cell lung cancer [J]. Lung Cancer. 2017;106:1-7.

97. Weide B, Martens A, Hassel JC, et al. Baseline biomarkers for outcome of melanoma patients treated with Pembrolizumab [J]. Clin Cancer Res. 2016 22(22):5487-96.

98. Martens A, Wistuba-Hamprecht K, Geukes Foppen M, et al. Baseline peripheral blood biomarkers associated with clinical outcome of advanced melanoma patients treated with Ipilimumab [J]. Clin Cancer Res. 2016; 22(12):2908-18.

99. Kazandjian D, Gong Y, Keegan P, et al. Prognostic value of the lung immune prognostic index for patients treated for metastatic non-small cell lung Cancer [J]. JAMA Oncol. 2019;5(10):1481-5.

100. Tietze JK, Angelova D, Heppt MV, et al. The proportion of circulating $\mathrm{CD} 45 \mathrm{RO}(+) \mathrm{CD} 8(+)$ memory $T$ cells is correlated with clinical response in melanoma patients treated with ipilimumab [J]. Eur J Cancer. 2017;75:268-79.

101. Subrahmanyam PB, Dong Z, Gusenleitner D, et al. Distinct predictive biomarker candidates for response to anti-CTLA-4 and anti-PD-1 immunotherapy in melanoma patients [J]. J Immunother Cancer. 2018;6(1):18.

102. Krieg C, Nowicka M, Guglietta S, et al. High-dimensional single-cell analysis predicts response to anti-PD-1 immunotherapy [J]. Nat Med. 2018;24(2): $144-53$

103. Liakou Cl, Kamat A, Tang DN, et al. CTLA-4 blockade increases IFNgammaproducing $\mathrm{CD} 4+\mathrm{ICOShi}$ cells to shift the ratio of effector to regulatory $\mathrm{T}$ cells in cancer patients [J]. Proc Natl Acad Sci U S A. 2008;105(39):14987-92.

104. Kulasinghe A, Perry C, Kenny L, et al. D-L1 expressing circulating tumour cells in head and neck cancers [J]. BMC Cancer. 2017;17(1):333.

105. Heitzer E, Ulz P, Geigl JB. Circulating tumor DNA as a liquid biopsy for Cancer [J]. Clin Chem. 2015;61(1):112-23.
106. Khagi Y, Goodman AM, Daniels GA, et al. Hypermutated Circulating Tumor DNA: Correlation with Response to Checkpoint Inhibitor-Based Immunotherapy[J]. Clin Cancer Res. 2017;23(19):5729-36.

107. Lee JH, Long GV, Boyd S, et al. Circulating tumour DNA predicts response to anti-PD1 antibodies in metastatic melanoma [J]. Ann Oncol. 2017;28(5): 1130-6.

108. Lee JH, Long GV, Menzies AM, et al. Association Between Circulating Tumor DNA and Pseudoprogression in Patients With Metastatic Melanoma Treated With Anti-Programmed Cell Death 1 Antibodies [J]. JAMA Oncol. 2018;4(5): 717-21.

109. Wang Z, Duan J, Cai S, et al. Assessment of blood tumor mutational burden as a potential biomarker for immunotherapy in patients with non-small cell lung Cancer with use of a next-generation sequencing Cancer gene panel [J]. JAMA Oncol. 2019;5(5):696-702.

110. Peters S, Cho BC, Reinmuth N, et al. Abstract CT074: Tumor mutational burden (TMB) as a biomarker of survival in metastatic non-small cell lung cancer (mNSCLC): Blood and tissue TMB analysis from MYSTIC, a Phase III study of first-line durvalumab \pm tremelimumab vs chemotherapy. Presented at: American Association for Cancer Research Annual Meeting 2019. Atlanta, $\mathrm{GA} ; 2019$.

111. Chen G, Huang AC, Zhang W, et al. Exosomal PD-L1 contributes to immunosuppression and is associated with anti-PD-1 response [J]. Nature. 2018;560(7718):382-6.

112. Del Re M, Marconcini R, Pasquini G, et al. PD-L1 mRNA expression in plasma-derived exosomes is associated with response to anti-PD-1 antibodies in melanoma and NSCLC [J]. Br J Cancer. 2018;118(6):820-4.

113. Gong B, Kiyotani K, Sakata S, et al. Secreted PD-L1 variants mediate resistance to PD-L1 blockade therapy in non-small cell lung cancer [J]. J Exp Med. 2019;216(4):982-1000.

114. Nakamura Y. Biomarkers for Immune Checkpoint Inhibitor-Mediated Tumor Response and Adverse Events [J]. Front Med (Lausanne). 2019;6:119.

115. Bedikian AY, Johnson MM, Warneke $C L$, et al. Prognostic factors that determine the long-term survival of patients with unresectable metastatic melanoma [J]. Cancer Investig. 2008;26(6):624-33.

116. Kelderman $\mathrm{S}$, Heemskerk $B$, van Tinteren $H$, et al. Lactate dehydrogenase as a selection criterion for ipilimumab treatment in metastatic melanoma [J]. Cancer Immunol Immunother. 2014;63(5):449-58.

117. Taniguchi $Y$, Tamiya A, Isa SI, et al. Predictive factors for poor progressionfree survival in patients with non-small cell lung Cancer treated with Nivolumab [J]. Anticancer Res. 2017;37(10):5857-62.

118. Weber JS, Tang H, Hippeli L, et al. Serum IL-6 and CRP as prognostic factors in melanoma patients receiving single agent and combination checkpoint inhibition [J]. J Clin Oncol. 2019;37:100.

119. Sosa A, Lopez Cadena E, Simon Olive C, et al. Clinical assessment of immune-related adverse events [J]. Ther Adv Med Oncol. 2018;10: 1758835918764628.

120. Yuen KC, Liu LF, Gupta V, et al. High systemic and tumor-associated IL-8 correlates with reduced clinical benefit of PD-L1 blockade [J]. Nat Med. 2020:26(5):693-8.

121. Schalper KA, Carleton M, Zhou M. Elevated serum interleukin-8 is associated with enhanced intratumor neutrophils and reduced clinical benefit of immune-checkpoint inhibitors [J]. Nat Med. 2020;26(5):688-92.

122. Conforti F, Pala L, Bagnardi V, et al. Cancer immunotherapy efficacy and patients' sex: a systematic review and meta-analysis [J]. Lancet Oncol. 2018; 19(6):737-46.

123. Wu Y, Ju Q, Jia K, et al. Correlation between sex and efficacy of immune checkpoint inhibitors (PD-1 and CTLA-4 inhibitors)[J]. Int J Cancer. 2018; 143(1):45-51.

124. Fulop T, Larbi A, Kotb R, et al. Aging, immunity, and cancer [J]. Discov Med. 2011;11(61):537-50

125. Kugel CH 3rd, Douglass SM, Webster MR, et al. Age correlates with response to anti-PD1, reflecting age-related differences in Intratumoral effector and regulatory T-cell populations [J]. Clin Cancer Res. 2018;24(21):5347-56.

126. Nishijima TF, Muss HB, Shachar SS, et al. Comparison of efficacy of immune checkpoint inhibitors (ICls) between younger and older patients: a systematic review and meta-analysis [J]. Cancer Treat Rev. 2016;45:30-7

127. Felip E, Ardizzoni A, Ciuleanu T, et al. CheckMate 171: a phase 2 trial of nivolumab in patients with previously treated advanced squamous nonsmall cell lung cancer, including ECOG PS 2 and elderly populations [J]. Eur J Cancer. 2020;127:160-72. 
128. Dudnik E, Moskovitz M, Daher S, et al. Effectiveness and safety of nivolumab in advanced non-small cell lung cancer: the real-life data [J]. Lung Cancer. 2018;126:217-23.

129. Murphy WJ, Longo DL. The surprisingly positive association between obesity and Cancer immunotherapy efficacy [J]. Jama. 2019;321(13):1247-8.

130. Wang Z, Aguilar EG, Luna Jl, et al. Paradoxical effects of obesity on T cell function during tumor progression and PD-1 checkpoint blockade [J]. Nat Med. 2019;25(1):141-51.

131. Baumann BC, Mitra N, Harton JG, et al. Comparative effectiveness of proton vs photon therapy as part of concurrent Chemoradiotherapy for locally advanced Cancer [J]. JAMA Oncol. 2019;6(2):237-46.

132. Routy B, Le Chatelier E. Gut microbiome influences efficacy of PD-1-based immunotherapy against epithelial tumors [J]. Science. 2018;359(6371):91-7.

133. Gopalakrishnan V, Spencer CN. Gut microbiome modulates response to anti-PD-1 immunotherapy in melanoma patients [J]. Science. 2018; 359(6371):97-103.

134. Chaput N, Lepage $P$, Coutzac C, et al. Baseline gut microbiota predicts clinical response and colitis in metastatic melanoma patients treated with ipilimumab[J]. Ann Oncol. 2019;30(12):2012.

135. Matson V, Fessler J, Bao R. The commensal microbiome is associated with anti-PD-1 efficacy in metastatic melanoma patients [J]. Science. 2018; 359(6371):104-8.

136. Sivan A, Corrales L, Hubert N, et al. Commensal Bifidobacterium promotes antitumor immunity and facilitates anti-PD-L1 efficacy [J]. Science. 2015; 350(6264):1084-9.

137. Parham P, Ohta T. Population biology of antigen presentation by MHC class I molecules [J]. Science. 1996;272(5258):67-74.

138. Havel JJ, Chowell D, Chan TA. The evolving landscape of biomarkers for checkpoint inhibitor immunotherapy [J]. Nat Rev Cancer. 2019;19(3):133-50.

139. Chowell D, LGT M. Patient HLA class I genotype influences cancer response to checkpoint blockade immunotherapy [J]. Science. 2018;359(6375):582-7.

140. Kvistborg P, Yewdell JW. Enhancing responses to cancer immunotherapy [J]. Science. 2018;359(6375):516-7.

141. McGranahan N, Rosenthal R, Hiley CT, et al. Allele-Specific HLA Loss and Immune Escape in Lung Cancer Evolution[J]. Cell. 2017;171(6):1259-71. e1211.

142. Theodoraki MN, Yerneni SS, Hoffmann TK, et al. Clinical significance of PDL1(+) Exosomes in plasma of head and neck Cancer patients [J]. Clin Cancer Res. 2018;24(4):896-905.

143. Yang JC, Hughes M, Kammula U, et al. Ipilimumab (anti-CTLA4 antibody) causes regression of metastatic renal cell cancer associated with enteritis and hypophysitis [J]. J Immunother. 2007;30(8):825-30.

144. Teraoka S, Fujimoto D, Morimoto T, et al. Early Immune-Related Adverse Events and Association with Outcome in Advanced Non-Small Cell Lung Cancer Patients Treated with Nivolumab: A Prospective Cohort Study [J]. J Thorac Oncol. 2017;12(12):1798.

145. Judd J, Zibelman M, Handorf E, et al. Immune-related adverse events as a biomarker in non-melanoma patients treated with programmed cell death 1 inhibitors [J]. Oncologist. 2017;22(10):1232-7.

146. Fujisawa Y, Yoshino K, Otsuka A, et al. Retrospective study of advanced melanoma patients treated with ipilimumab after nivolumab: analysis of 60 Japanese patients [J]. J Dermatol Sci. 2018;89(1):60-6.

147. Kim HI, Kim M, Lee SH, et al. Development of thyroid dysfunction is associated with clinical response to PD-1 blockade treatment in patients with advanced non-small cell lung cancer [J]. Oncoimmunology. 2017;7(1): e1375642

148. Teulings HE, Limpens J, Jansen SN, et al. Vitiligo-like depigmentation in patients with stage III-IV melanoma receiving immunotherapy and its association with survival: a systematic review and meta-analysis [J]. J Clin Oncol. 2015;33(7):773-81.

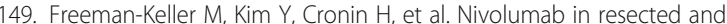
Unresectable metastatic melanoma: characteristics of immune-related adverse events and association with outcomes [J]. Clin Cancer Res. 2016: 22(4):886-94.

150. Rzepecki AK, Cheng H, McLellan BN. Cutaneous toxicity as a predictive biomarker for clinical outcome in patients receiving anticancer therapy [J]. J Am Acad Dermatol. 2018;79(3):545-55.

151. Shukla SA, Bachireddy P, Schilling B, et al. Cancer-Germline Antigen Expression Discriminates Clinical Outcome to CTLA-4 Blockade [J]. Cell. 2018, 173(3):624-33.e8.
152. Rodig SJ, Gusenleitner D. MHC proteins confer differential sensitivity to CTLA-4 and PD-1 blockade in untreated metastatic melanoma [J]. Sci Transl Med. 2018;10(450):eaar3342.

153. Rizvi H, Sanchez-Vega F, La K, et al. Molecular determinants of response to anti-programmed cell death (PD)-1 and anti-programmed death-ligand 1 (PD-L1) blockade in patients with non-small-cell lung Cancer profiled with targeted next-generation sequencing [J]. J Clin Oncol. 2018;36(7):633-41.

154. Althammer S, Tan TH, Spitzmüller A, et al. Automated image analysis of NSCLC biopsies to predict response to anti-PD-L1 therapy [J]. J Immunother Cancer. 2019;7(1):121.

155. Yu Y, Zeng D, Ou Q, et al. Association of Survival and Immune-Related Biomarkers with Immunotherapy in patients with non-small cell lung Cancer: a meta-analysis and individual patient-level analysis [J]. JAMA Netw Open. 2019;2(7):e196879.

156. Cristescu R, Mogg R, Ayers M, et al. Pan-tumor genomic biomarkers for PD-1 checkpoint blockade-based immunotherapy[J]. Science. 2018;362(6411): eaar3593.

157. Balachandran VP, Łuksza M, Zhao JN, et al. Identification of unique neoantigen qualities in long-term survivors of pancreatic cancer [J]. Nature. 2017:551(7681):512-6.

158. Sarkizova S, Hacohen N. How T cells spot tumour cells [J]. Nature. 2017; 551(7681):444-6.

159. Jiang P, Gu S, Pan D, et al. Signatures of T cell dysfunction and exclusion predict cancer immunotherapy response [J]. Nat Med. 2018:24(10):1550-8.

\section{Publisher's Note}

Springer Nature remains neutral with regard to jurisdictional claims in published maps and institutional affiliations.
Ready to submit your research? Choose BMC and benefit from:

- fast, convenient online submission

- thorough peer review by experienced researchers in your field

- rapid publication on acceptance

- support for research data, including large and complex data types

- gold Open Access which fosters wider collaboration and increased citations

- maximum visibility for your research: over $100 \mathrm{M}$ website views per year

At BMC, research is always in progress.

Learn more biomedcentral.com/submission 